\title{
Estimating shallow shear velocities with marine multi-component seismic data
}

\author{
Michael H. Ritzwoller and Anatoli L. Levshin \\ Center for Imaging the Earth's Interior, University of Colorado at Boulder, \\ Boulder, CO 80309-0390 \\ (303)492-7075; ritzwoller@ciei.colorado.edu
}

\begin{abstract}
Accurate models of shear velocities in the shallow subsurface $(<300 \mathrm{~m}$ depth beneath the sea floor) would help to focus images of structural discontinuities constructed, for example, with $P$ to $S$ converted phases in marine environments. Although multi-component marine seismic data hold a wealth of information about shear velocities from the sea floor to depths of hundreds of meters, this information remains largely unexploited in oil and gas exploration offshore. We present a method, called the Multi-Wave Inversion (MWI) method, that utilizes the full richness of information in marine seismic data. At present, MWI jointly uses the observed travel times of $P$ and $S$ refracted waves, the group and phase velocities of fundamental and first overtone interface waves, and the group velocities of guided waves to infer shear velocities and $V_{p}: V_{s}$ ratios. We show how to obtain measurements of the travel times of these diverse and in some cases dispersive waves and how they are utilized in the MWI method to estimate shallow shear velocities. We illuminate the method with examples of marine data acquired by Fairfield Industries, Unocal, and Western Geophysical and apply MWI to the Fairfield Industries data to obtain a model of $V_{s}$ with uncertainties to a depth of $225 \mathrm{~m}$ and $V_{p}: V_{s}$ to about $100 \mathrm{~m}$ depth. We conclude by discussing the design of offshore surveys necessary to provide information about shallow shear velocity structures with a particular cautionary note sounded about the height of the acoustic source above the seafloor.
\end{abstract}

Submitted to Geophysics, June 21, 2000. 


\section{INTRODUCTION}

The ability to construct reliable models of the shear velocities of marine sediments down to $100 \mathrm{~m}$ or more beneath the sea floor is important in a number of disparate disciplines. For example, for exploration seismologists these models would help to improve the shear wave static correction needed in oil and gas exploration (e.g., Mari, 1984; Marsden, 1993). This need has grown in importance as multi-component marine surveys have become common (e.g., Caldwell, 1999). Images of deep shear velocity horizons are sometimes better than those achievable with $P$-waves particularly in and around gas clouds (e.g., Zhu et al., 1999) and beneath high velocity layers (e.g., Purnell, 1992) and together with $P$-waves provide Poisson's ratio which is used as a proxy for porosity (e.g., Hamilton, 1979; Gaiser, 1996). For geotechnical engineers these models would help to constrain the shear modulus for investigations of foundation vibrations, slope instabilities, and expected earthquake effects (e.g., Smith, 1986; Frivik and Hovem, 1995, Stokoe et al., 1999). Also, knowledge of sedimentary acoustic properties is needed to understand acoustic wave loss which is important for sonar propagation, particularly in shallow water (e.g., Stoll et al., 1988).

Over the past few decades, the shear or geoacoustic properties of marine sediments have been extensively studied in the laboratory and in situ in a variety of marine environments from near the shore to the deep oceans (e.g., Bibee and Dorman, 1991). Akal and Berkson (1986), Rauch (1986), Stoll (1989), and Hovem et al. (1991) present reviews or collections of articles on aspects of the subject. The greatest advances appear to have been in the use of interface waves to estimate shallow shear velocities, which is perhaps ironic given the pains taken to filter out these waves in land surveys (e.g., Saatçilar and Canitez, 1988; Hermann and Russell, 1990; Shieh and Herrmann, 1990; Blonk, 1995; Ernst and Herman, 1998).

In a marine setting, the waves trapped near the solid-fluid interface are sometimes called Scholte waves (Scholte, 1958), in contrast with Stoneley waves near a solid- 
solid interface or Rayleigh waves near the air-solid interface. All of these waves, however, are dispersive with phase and group velocities that are sensitive primarily to shear velocities at depths that are inversely related to frequency. The methods of analysis fall into four general categories. First is the measurement of the velocities of the fundamental and first-overtone with multiple-filtering methods sometimes called frequency - time analyses (e.g., Dziewonski et al., 1969; Levshin et al., 1972, 1989; Cara, 1973). Recent studies include Dosso and Brooke (1995), Essen et al. (1998), and Kawashima et al. (1998). Second are differential multi-receiver or multi-source phase velocity measurements which have been applied primarily to the fundamental mode (e.g., recent studies include Park et al., 1999; Stokoe and Rosenblad, 1999; Xia et al., 1999). The third method is the measurement of the phase velocities of the fundamental and several overtones using $\omega-k$ analyses (e.g., Gabriels et al., 1987; Snieder, 1987). Finally, waveform fitting has also been applied in a few studies to estimate shear velocities and $Q$ simultaneously (e.g., Ewing et al., 1992; Nolet and Dorman, 1996). We note that modes higher than the first or second overtones are typically not interface waves, but may sum to generate guided waves each of which is trapped in a waveguide which may extend well below the interface (e.g., Kennett, 1984). Techniques for studying surface waves are also highly developed in regional and global seismology (e.g., Knopoff, 1972; Ritzwoller and Lavely, 1995; Ritzwoller and Levshin, 1998; Vdovin et al., 1999; and many others).

Each of the methods above has its strengths and weaknesses. Any technique that utilizes the fundamental and first-overtone exclusively will provide little information about shear velocities below a few tens of meters unless waves can be observed at very low frequencies (i.e., below $2 \mathrm{~Hz}$, which is uncommon in exploration or geotechnical seismic surveys). Waveform fitting methods require detailed knowledge of the amplitude and phase response of the instruments, information about instrument - sediment coupling, and a priori knowledge of or joint inversion for a $Q$ model. Finally, methods based on $\omega-k$ analyses are typically best suited for 1-D inversions and typical shot 
spacings in marine exploration may be too coarse for analysis of interface waves.

We describe a method that is not intimately dependent on knowledge of instrument responses or $Q$, is designed for application in multiple dimensions, and provides information below the top few tens of meters beneath the sea floor. The method is based on the joint inversion of interface wave and guided wave dispersion and body wave travel times. We call this method Multi-Wave Inversion (MWI). As presented here, MWI simultaneously interprets interface wave group and phase velocities, the group velocities of guided waves, and refracted $S$ travel times, but MWI is generalizable to other wave types such as multiple $S$ bounce phases and converted phases.

We follow the common practice of interpreting the dispersion of interface waves in terms of the normal modes of the medium of propagation. (The Appendix presents a summary.) Normal mode eigenfunctions give the particle motion of the waves, phase and group velocities may be computed from the eigenfrequencies and their frequency dependence, and it is also a simple matter to compute integral sensitivity kernels (e.g., Rodi et al., 1975). Guided waves are also a dispersive wave but, as we describe below, are not as naturally interpreted from a modal perspective because each guided wave typically comprises more than a single mode. This characteristic of guided waves renders the inverse problem non-linear and we discuss how to deal with this non-linearity. We model the refracted $S$ waves using ray theory.

The MWI method is applied to a small data set provided to the authors by Fairfield Industries. These four-component data are recorded in very shallow water $(\sim 5 \mathrm{~m})$ off Louisiana. There are three receivers spaced at about $1 \mathrm{~km}$, and several hundred shots spaced about every $25 \mathrm{~m}$. The depth of the model is determined by the maximum range to which refracted $S$ is observed. Shear waves are unambiguously observed to about $1.2 \mathrm{~km}$ distance, which means the $S$ model extends to a little more than $200 \mathrm{~m}$ beneath the sea floor. $P$ travel times are measured to the same distance and because $P$ turns above $S$, the $P$ model extends only to a depth of about $100 \mathrm{~m}$. Due to this source - receiver geometry, we present results from our Monte-Carlo inversion only 
for a 1-D model, but MWI is applicable in multiple dimensions. Indeed, we provide evidence for strong variability of interface wave dispersion over the region of study.

Future enhancements and extensions of MWI include generalizing the inversion to multiple dimensions and investigating its application to land data. MWI, as we describe, is appropriate for any medium in which the heterogeneities are sufficiently smooth so as not to strongly scatter the interface, guided, and refracted waves. Marine environments characterized by active sedimentary deposition tend to display such characteristics, but so do some land settings with particularly strong "ground-roll" (e.g., Al-Husseini et al., 1981). In addition, if the detailed information needed to perform waveform fitting exists, MWI would provide a very good starting model for waveform inversion. Although we regard waveform inversion to be a desirable

direction for the future development of the MWI method, it remains to be seen if it will provide superior results to a method based purely on travel times and wave dispersion.

The remainder of the paper is divided into four sections. In the section entitled 'Theoretical Expectations', we present a discussion of the physics of waves in a marine environment. In the next section, called 'Data and Measurement', we describe the data and measurements used in this study. In the fourth section of the paper, titled 'Inversion', we present the use of MWI to estimate a 1-D $S$ model and assess its uncertainty. Finally, we conclude with a discussion of the specifications of a marine survey designed to provide the information needed for the MWI method.

\section{THEORETICAL EXPECTATIONS}

Synthetic experiments have proven useful to gain insight into the nature of the various wave types and phases that appear in multi-component marine data. The purpose of this section is to use synthetic wave-fields constructed using the shallow marine model discussed in the inversion section of the paper (i.e., Fig. 17) to identify 
the main phases and wave types expected in a marine survey and to guide the use of the MWI method to infer shallow shear velocity structure. The wave types that we identify as potentially useful include phase and group velocity dispersion measurements of fundamental and first overtone interface waves, group velocities of guided waves, refracted $S$ and $P$ travel times, differential travel times for refracted multiple bounce phases (e.g., $S-S S$, etc.), and travel times of $P$ to $S$ converted phases.

The appendix provides a brief summary of the use of normal modes to compute synthetic seismograms in simple plane layered media. For the synthetic seismograms shown in this section, we have summed the first 11 Rayleigh modes (the fundamental and the first 10 overtones) from 1 to $15 \mathrm{~Hz}$, tapering the resultant spectrum from 1 - $1.5 \mathrm{~Hz}$ and 12 - $15 \mathrm{~Hz}$. The shallow marine shear model has a fluid layer overlying a vertically layered half space. An explosive source with a characteristic time of $10^{-3}$ $\mathrm{s}$ is placed $2 \mathrm{~m}$ above the ocean floor. Without lateral heterogeneities or anisotropy, Love waves will not be generated by an acoustic source in a fluid region. Thus, Love waves are not included in the synthetic seismograms shown here and all horizontal components are directed radially away from the source. The shear $Q$ model is frequency independent and approximately constant with depth with an average value of 23 and physical dispersion is included in the elastic moduli.

To illustrate modal summation, Figure 1 presents a horizontal displacement seismogram in which each of the modal contributions is plotted separately. Each higher mode contributes successively higher group velocities, but the amplitudes reduce systematically so that the entire seismogram is very well approximated by the first seven modes. The frequency content of the seismograms is determined by the $Q$ model and the height of the acoustic source above the sea floor.

Figure $2 \mathrm{a}$ and $2 \mathrm{~b}$ display the excitation spectra of the fundamental mode for source heights ranging from $1 \mathrm{~m}$ to $50 \mathrm{~m}$ above the sea floor for two different subsurface models. In the Appendix, the excitation spectrum is denoted $A(\omega, h)$ and source height is $d-h$ where $d$ is the depth of the water layer and $h$ is the depth of the 
source $(d>h)$. Figure 2a was constructed with the marine model displayed in Figure 17. A source height of $3 \mathrm{~m}$ with this model produces an excitation spectrum that is flat from $3-10 \mathrm{~Hz}$. As the source is raised above the sea floor the excitation of the fundamental mode is reduced systematically. The spectrum also becomes more narrow banded and peaks at successively lower frequencies. The details of these excitation curves depend strongly on the subsurface model. In particular, as the shear velocities in the near surface sediments increase, the efficiency of conversion from acoustic energy in the water column to shear energy in the sediments improves. Figure $2 \mathrm{~b}$ shows that if the shear velocities are multiplied by a factor of two then to achieve a specific excitation, the source can be further off the sea floor than for the model with lower shear velocities. Finally, Figure 2c displays the excitation spectra for the fourth overtone using the marine model in Figure 17. The excitation of the overtones decays with increasing source height slower than the fundamental modes. The consequences for the design of marine surveys are discussed in a separate section near the end of the paper.

All of the modes that contribute to Figure 1 are dispersive; that is their phase and group velocities are not equal. The group and phase velocity curves for our shallow marine model are displayed in Figure 3. The amplitudes of the resultant waves will be expected to maximize near the Airy phases, that is near the local maxima and minima of the group velocity curves. Strong arrivals are, therefore, expected at frequencies above $\sim 2 \mathrm{~Hz}$ for the fundamental mode and above $5 \mathrm{~Hz}$ for the first overtone. As Figure 4 shows, the displacement eigenfunctions of the fundamental and first overtone at these frequencies maximize near the sea floor and are trapped in the top $10-20 \mathrm{~m}$ beneath the sea floor. These waves are, therefore, referred to as interface waves and we identify them as $I_{0}$ and $I_{1}$, respectively. Different modes that have nearly the same group velocities and similar phase velocities will sum coherently and may generate a large amplitude arrival. For our shallow marine model, the Airy phases from the first $(\sim 3 \mathrm{~Hz})$, second $(\sim 4-6 \mathrm{~Hz})$, third $(\sim 8-9 \mathrm{~Hz})$, and higher overtones create 
a plateau or ridge on the group velocity diagram and sum to produce a wave guide arrival that we call a guided wave and denote as $G_{1}$. There may be several guided waves trapped in different waveguides each creating a separate ridge on the group velocity diagram. We also identify a wave labeled $G_{2}$ that is composed of the fourth and fifth overtones. Figure $4 \mathrm{c}$ demonstrates that $G_{1}$ is trapped in about the top 70 $m$ beneath the sea floor. Thus, the interface and guided waves provide information about different depth ranges of the subsurface. This fact is exploited by the MWI method.

Figure 4 further demonstrates that the polarization of each mode is frequency dependent. Two good rules of thumb follow. First, the fundamental mode is approximately vertically polarized above $5 \mathrm{~Hz}$, is nearly circularly polarized at $3 \mathrm{~Hz}$, and is horizontally polarized at $2 \mathrm{~Hz}$. Second, the overtones are all nearly horizontally polarized at the sea floor except at very low frequencies. Thus, the fundamental is observable on both vertical and horizontal components, but the other modes are poorly observed on vertical records except at frequencies below about $3 \mathrm{~Hz}$.

These expectations are confirmed by the synthetic record sections displayed in Figure 5. We wish to explore how information extractable from these waveforms can be used to infer shallow subsurface $S$ structure. The inverse problem using measurements of group and phase velocity has been well studied. Given the full set of theoretical dispersion curves presented in Figure 3, the input model could be very accurately reconstructed. These curves, however, cannot be fully estimated from the data.

Dispersion measurements are commonly obtained using frequency - time analysis (e.g., Dziewonski et al., 1969; Levshin et al., 1972, 1989; Cara, 1973). An example is shown in Figure 6. Broad-band $(\sim 2-8 \mathrm{~Hz})$ dispersion measurements of the fundamental interface wave are obtainable on both the vertical and horizontal components. Dispersion measurements for the first overtone interface wave are also possible on the horizontal component from about $5-8 \mathrm{~Hz}$. Note that for the higher modes the largest 
amplitudes are, indeed, near the Airy phases. Thus, broad-band dispersion curves for the higher modes would be difficult to obtain even on synthetic data. The best that can be done in practice is to measure the velocity of each guided wave on the horizontal component. These velocities then define the associated ridges or plateaus in the group velocity diagram; e.g., the first through third overtones for $G_{1}$ seen in Figure 6 .

In summary, these synthetic experiments indicate that one should be able to measure and interpret the group and phase velocities of $I_{0}$ and $I_{1}$ and, potentially, the group velocity of any guided wave that exists in the data. Inspection of the vertical component frequency - time diagram in Figure 6 also suggests that a group velocity measurement can be made for the first overtone at very low frequencies $(\sim 2.5$ $\mathrm{Hz}$ ). This may, indeed, be the case in some marine surveys, but in the next section we show that this feature does not appear in the Fairfield Industries data, probably due to the effects of the instrument response and attenuation.

The remaining information in the synthetic data is more fruitfully considered from a body wave perspective. Observations of the travel times of refacted $S$ are relatively easy to make on horizontal component marine data as we show in section of of the paper on data and measurements. In addition, as Figure 7 shows, these measurements complement interface and guided wave data by providing sensitivity to structures deeper than the 50 - $75 \mathrm{~m}$ that guided waves sample. As discussed in the next section, $S$ travel times may be obtained from the Fairfield Industries data to a range of only about $1.2 \mathrm{~km}$, which means that the shallow $S$ model can extend no deeper than about $225 \mathrm{~m}$ (see Fig. 7). The double and triple refracted surface bounce phases $S S$ and $S S S$ are also apparent in the synthetic wave-field in Figure 5, with each bounce phase emerging successively further from the source. Measurements of the absolute travel times of these phases are difficult to obtain, but global scale studies have shown that the differential times $S S-S, S S S-S$, and $S S S-S S$ can be measured robustly and interpreted, with some caveats, in a straightforward way (e.g., Woodward and 
Masters, 1991). This is done by cross-correlating the earlier arriving of each phase pair with the later arrival. In doing so all phase shifts resulting from the surface bounce(s) are corrected by Hilbert transforming. Finally, the converted phase that we call $P d S$ is also useful to help constrain the depths of jump discontinuities. This phase is a down-going $P$ wave that converts to an up-going $S$ wave at a discontinuity at a depth of $d$ meters below the sea floor; e.g., a down-going $P$ that converts to an up-going $S$ at $70 \mathrm{~m}$ depth is called $P 70 S$. PdS phases are dominantly expected to precede $S$ and follow $P$ in the waveform, as Figure 8 shows.

\section{DATA AND MEASUREMENTS}

The discussion in this and the subsequent section will focus on measurements and interpretation of a small data set provided to us by Fairfield Industries. Figure 9 displays the experimental layout in which a line of shots is recorded at three receivers. The data divides naturally into three receiver gathers and each gather separates into shots on either side (northing $(\mathrm{N})$ or southing $(\mathrm{S})$ ) of the receiver: lines $1 \mathrm{~N}, 1 \mathrm{~S}, 2 \mathrm{~N}$, $2 \mathrm{~S}, 3 \mathrm{~N}$, and $3 \mathrm{~S}$. Shots occur in about $5 \mathrm{~m}$ of water, so source heights average about 3 $m$ above the sea floor. As discussed further in the section of the paper on designing marine surveys, it is the proximity of the shots to the sea floor that makes these data so useful to infer shallow shear velocity structure.

Figure 10 presents examples of vertical and horizontal record sections. The main wave types in evidence in the observed wave-field are similar to those of the synthetic wave-field in Figure 5. The major difference between the observed and synthetic wave-fields is in the amplitudes and frequency content. Improving agreement in these quantities will require a knowledge of the amplitude response of the instruments and a model of $Q$ which may be frequency dependent.

We measure the fundamental and first-overtone dispersion curves from vertical

and horizontal component data, respectively. Figure 11 displays an example of the 
frequency - time diagrams used for these measurements. The guided wave $G_{1}$ is observed between 4 and $9 \mathrm{~Hz}$ and averages about $140 \mathrm{~m} / \mathrm{s}$, and $G_{2}$ is observed between about 6 and $9 \mathrm{~Hz}$ and averages about $170 \mathrm{~m} / \mathrm{s}$. Refracted $S$ travel times are also measurable as Figure 12 shows.

The wave-paths of some of the measurements, referenced to Figure 9, are shown in Figure 13. Fundamental group velocity measurements are obtained from source - receiver distances ranging from about $75 \mathrm{~m}$ to $650 \mathrm{~m}$. The maximum distance is limited by the length of the observed time series (16 s). Consequently, the first overtone, being faster than the fundamental, is measured to greater distances, out to about $1 \mathrm{~km}$ in some cases. The locations of the $S$ travel time measurements are not shown in Figure 13, but are typically obtained at source - receiver distances ranging from about $100 \mathrm{~m}$ up to $1.5 \mathrm{~km}$. Observations past about $1.2 \mathrm{~km}$ become difficult with this data set. This distance range is reflected in the standard deviations of the measured $S$ travel times shown in Figure 15.

The phase velocities of the dispersed waves are also potential observables. Although phase and group velocities are simply related through a frequency derivative, the group velocity measurements are obtained on the envelope of the waveform whereas phase velocity estimates derive from observations of the phases themselves. Thus, although phase and group velocity measurements are not completely independent, it is useful to measure them both. Observations of phases are defined unambiguously only modulo $2 \pi$, so there is an inherent ambiguity in the phase velocity inferred from the observed phase at a given source - receiver distance. This problem worsens as the source - receiver distance increases. One way around this problem is to measure the difference in the observed phase for two paths that are nearly coincident so that the expected phase difference between the two observations is much smaller than $2 \pi$. We measure differential phases for shots that are nearly the same distance from a particular receiver. These differential phase velocity measurements constrain the phase velocity of the wave only between the shots and, therefore, pro- 
vide higher horizontal spatial resolution than the absolute velocity measurements. This is reflected in Figure 13 which shows that the phase velocity measurements all have shorter baselines and do not terminate at the receiver.

The average of the velocities or travel times of several wave types is shown in Figure 14. The standard deviations of the measurements about these means are shown in Figure 15. The standard deviations of the $G_{1}$ and $G_{2}$ measurements also vary with frequency. In the middle of both of the $G_{1}$ and $G_{2}$ curves the standard deviation is small $(\sim 5 \mathrm{~m} / \mathrm{s}, \sim 3 \%)$ but more than doubles near the end-points. The guided wave measurements are somewhat more difficult to interpret than other dispersion measurements because they are composed of several modes and their appearance on frequency-time images is variable. We discuss the implications of this for inversion in the next section.

Although we mentioned in the section on theoretical expectations that differential $S$ travel time measurements can be obtained and the $S S$ and $S S S$ phases are quite apparent in the observed record section shown in Figure 10, we do not use these phases here and this is left as a direction for future work.

\section{INVERSION}

Based on the source height above the sea floor (discussed further in the next section), the Fairfield Industries data are particularly amenable to inversion for shallow shear velocity structure. These data are distributed approximately linearly in twodimensions (2-D), as seen in Figure 9. Thus, a 3-D inversion is out of the question

and, with receivers spaced about $1 \mathrm{~km}$ apart, it is not even possible to do a meaningful 2-D inversion because subsurface shear velocities vary on much smaller scales than the receiver spacing. Thus, the best we can do here is an inversion of the average measurements for a 1-D model. With a suitable distribution of sources and receivers, however, the MWI method is entirely suited for 2-D or 3-D inversions. 
To investigate the spatial variability of the measurements prior to inversion, we use the 115 fundamental mode group velocity dispersion curves obtained for the wave paths shown in Figure 13a to produce an estimate of the smooth spatial variation in group velocities. This is an example of surface wave tomography (e.g., Barmin et al., 2000) and a similar approach was taken by Stoll et al. (1994b). We obtain Figure 16 which shows that group velocities are considerably slower toward the 'north' by up to $25 \%$ relative to the far 'south'. The relative error of most of the measurements is presented in Figure 15. This smooth spatial variation of fundamental mode group velocities reduces the relative error in the measurements from about 6-9\% to under $2 \%$ on average. Therefore, most of the variability in the measurements is systematic and we conclude that measurement variance dominantly has a structural cause. A 2-D model would be able to fit the measurements considerably better than the 1-D model whose construction we describe here.

We invert six curves that represent the average of all measurements for a 1-D model, the five dispersion curves and one travel time curve shown in Figure 14. These include the group velocities of the fundamental and first overtone interface waves and two guided waves $\left(G_{1}, G_{2}\right)$, the phase velocity of $I_{0}$, and the travel time curve of the refracted $S$ wave.

As discussed briefly in the preceding section, each guided wave is composed of a different combination of modes. This renders these waves more difficult to interpret in practice than the dispersion measurements for a single mode. For this reason, we do not attempt to fit the dispersion curves of $G_{1}$ and $G_{2}$ directly but only to fit certain discrete velocities along each average group velocity curve. For $G_{1}$ we use the velocities at 5.5 and $7.0 \mathrm{~Hz}(\sim 130 \mathrm{~m} / \mathrm{s}$ and $\sim 140 \mathrm{~m} / \mathrm{s})$, which we interpret as the second and third overtones, respectively. For $G_{2}$ we only use the velocity at 7.0 $\mathrm{Hz}(\sim 170 \mathrm{~m} / \mathrm{s})$ which we interpret as the fourth overtone. The assignment of the modal constitution of the guided waves requires insight into the general dispersion characteristics of the medium. This insight must be based on a fairly good subsurface 
$S$ model prior to the introduction of the guided waves into the inversion. In practice, the guided waves must be introduced after models that fit the other data are found. This renders the inversion formally non-linear because the interpretation of the guided waves depends on the models that fit the other data. Thus, guided waves are only useful in the context of an inversion with other more simply interpreted data, such as the refracted $S$ and interface dispersion measurements here, or a priori information about subsurface structure (e.g., from bore-hole measurements).

Inversions can be performed with any of a variety of different methods. We choose a Monte Carlo method because it is simple and provides several advantages. First, it permits the application of a wide variety of side constraints on the model. Second, it presents a range of models as acceptable which allows uncertainty estimates to be assigned to the estimated model. These estimates, however, are defined relative to the relative weighting assigned to the different data types in the penalty function and the side constraints to which the model must adhere. Finally, it is easy to add new data as they become available which allows the model to develop iteratively.

We parameterize each model as a stack of about 33 constant velocity layers that extend from the sea floor to a depth of about $300 \mathrm{~m}$ underlying the water layer. Layer thicknesses grow gradually with depth from $1.2 \mathrm{~m}$ directly below the sea floor to $\sim 16$ $\mathrm{m}$ in the lowermost layer. Each model is a perturbation to a starting model that we constructed by explicitly inverting the group velocity curves of the fundamental mode and the first overtone for a shallow model down to a depth of about $30 \mathrm{~m}$ below the sea floor, fixing the top $30 \mathrm{~m}$ at this model, and then inverting the $S$ travel times for a model from $30 \mathrm{~m}$ to $300 \mathrm{~m}$ depth. This model appears sufficient to identify the modal constitution of the guided waves. The allowable perturbations in each layer are uniformly distributed in a wide band about this starting model such that the resultant model exhibits monotonically increasing velocities with depth. To avert unnecessarily oscillatory models, some kind of 'smoothing constraint' on the allowable models is necessary in the absence of explicit a priori information about the location 
of low velocity layers. We choose the monotonicity constraint because there is little indication of shadow zones in the $S$ arrivals. If shadow zones were to exist, then it would be reasonable to allow low velocity zones in the appropriate depth ranges.

The penalty function that defines the set of acceptable models is based solely on total $\chi^{2}$ misfit. This statistic divides into the misfit to the measurements of single mode dispersion $\left(\chi_{\text {disp }}^{2}\right), S$ travel times $\left(\chi_{\mathrm{S}}^{2}\right)$, and the discrete guided wave velocities $\left(\chi_{\mathrm{gW}}^{2}\right)$ as follows:

$$
\begin{aligned}
\chi^{2} & =\chi_{\text {disp }}^{2}+\chi_{\mathrm{S}}^{2}+\chi_{\mathrm{gw}}^{2} \\
\chi_{\text {disp }}^{2} & =\sum_{n}^{N} \frac{1}{\Delta \omega_{n}} \int \sigma_{n}^{-2}(\omega)\left[U_{n}(\omega)-\hat{U}_{n}(\omega)\right]^{2} d \omega \\
\chi_{\mathrm{S}}^{2} & =\frac{1}{\Delta \ell} \int \sigma_{T}^{-2}(\ell)[T(\ell)-\hat{T}(\ell)]^{2} d \ell \\
\chi_{\mathrm{gw}}^{2} & =M^{-1} \sum_{m}^{M} \sigma_{m}^{-2}\left[U_{m}(\omega)-\hat{U}_{m}(\omega)\right]^{2}
\end{aligned}
$$

where $\omega$ is frequency, $\ell$ is distance, $U$ represents a measured velocity, $T$ is a measured travel time, $\hat{U}$ and $\hat{T}$ are quantities predicted by a model, $n$ is the dispersion measurement index, $m$ is the index for the discrete guided wave velocities, $\sigma_{n}$ represents the frequency dependent standard deviation of dispersion measurement $n, \sigma_{T}$ is the distance dependent standard deviation of the $S$ travel time, and the integrals are performed over the limits of the measurements of width $\Delta \omega_{n}$ for dispersion measurement $n$ and $\Delta \ell$ for the travel time.

The ensemble of acceptable models is defined by a total $\chi^{2}<4$, which means that the data on average are fit to twice the standard deviation of the measurements (see Fig. 15). We considered several hundred thousand trial models from which about 300 'winners' emerged. One of the better fitting models, shown in Figure 17, is the model we use as a reference throughout the paper. $S$ velocities range from about $45 \mathrm{~m} / \mathrm{s}$ at the sea floor to nearly $500 \mathrm{~m} / \mathrm{s}$ at a depth of $225 \mathrm{~m}$. The depth gradient is very high in the top $20 \mathrm{~m}$, as expected for compacting marine sediments (e.g., Hamilton, 1980; Meissner et al., 1985), and between depths of $40 \mathrm{~m}$ and $80 \mathrm{~m}$, but generally decreases 
with depth. The high gradient regions are modeled with prominent discontinuities between about 10 and $20 \mathrm{~m}$ and at a depth of about $70 \mathrm{~m}$. The $P$ model in Figure $17 \mathrm{~b}$ is estimated independently from the $S$ model using the measured refracted $P$ travel times with the constraints that the velocity at the sea floor is the acoustic velocity in sea water and that $P$ velocity also increases monotonically with depth. Like the $S$ model, the $P$ model exhibits prominent jump discontinuities between $15 \mathrm{~m}$ and 20 $\mathrm{m}$ depth and at a depth of about $70 \mathrm{~m}$. As shown in Figure 7, at a given source receiver distance $P$ turns at about half the depth of $S$. Because we obtained $P$ travel times on the same records as $S$ and, therefore, only to a distance of about $1.4 \mathrm{~km}$, the $P$ model extends only to about $100 \mathrm{~m}$ beneath the sea floor. $P$ travel times can be measured to much greater ranges than $S$ and the $P$ model is extendible to greater depths easily. The $V_{p}: V_{s}$ ratio, shown in Figure 17, is in excess of 20 in the shallow layers, decreases rapidly to a depth of about $20 \mathrm{~m}$ and then more gradually to attain a value of about 4 at a depth of $100 \mathrm{~m}$.

Figure 18a quantifies the range of acceptable models. The uncertainties are fairly conservative. We have used a $2 \sigma$ misfit criterion $\left(\chi^{2}<4\right)$ and have then plotted error bars that are twice the standard deviation across the acceptable models in each layer. Figure 18b displays the range of vertical shear wave travel times exhibited by the ensemble of models for each depth. There are no acceptable models that run systematically along the outskirts of the errors bars in Figure 18a. Rather an acceptable model may approach one end of an error bar at some depth, but then returns to more normal values at other depths. For this reason, the error bars on vertical travel times are much smaller than the uncertainties in the model $(<2 \%$ in half width compared with $\sim 7 \%$ at the bottom of the model). If a model is faster than average deep in the model it will be compensated by being slower than average higher up in the model. The use of a variety of wave types with differing depth sensitivities is designed to limit these trade-offs, but only the $S$ travel times constrain features below a depth of $\sim 70 \mathrm{~m}$ beneath the sea floor. Thus, these trade-offs occur mainly 
between $70 \mathrm{~m}$ and the bottom of the model. The error bars in the model and in the vertical travel time both are dominantly caused by variance in the data due to lateral structural inhomogeneity. Therefore, the uncertainties in these quantities would be substantially reduced with a 2-D inversion.

The importance of modeling $S$ trave times accurately for the shear static correction is seen by the fact that $S$ spends an inordinate amount of time in shallow layers in a marine environment: in this model about $750 \mathrm{~ms}$ in the top $250 \mathrm{~m}$ and nearly 200 $\mathrm{ms}$ in the top $25 \mathrm{~m}$ alone. Physically reasonable a priori uncertainties in the shear velocities in the top $25 \mathrm{~m}$ could translate to $\sim 100 \mathrm{~ms}$ receiver static prior to inversion.

The largest uncertainty in the model occurs around $70 \mathrm{~m}$ depth because the depth of the discontinuity near this point in the model is not unambiguously determined by the data we invert. All of the models in the ensemble do possess a significant discontinuity somewhere between 60 and $80 \mathrm{~m}$. The discontinuity that appears in the $P$ model is consistent with this depth range, but is similarly poorly constrained. In principle, we should be able to get more information from the phase $P d S$ which could help in localizing shallow discontinuities. The instrument response of the data should be deconvolved and, if possible, the phase should have an anti-attenuation filter applied prior to processing. Unfortunately, the instrument responses of the Fairfield Industries data are unknown to the authors and we have, to date, constructed only a very crude $Q$ model. We have, therefore, not included observations of $\operatorname{Pd} S$ in the inversion, but the raw waveforms can be used in an approximate way to test the location of the discontinuities in the model.

Figure 19 displays horizontal component seismograms with theoretical arrivals of the phase $P d S$ overplotted for a number of hypothetical reflector depths. The $P$ and $S$ velocities used to compute the theoretical arrival times for each hypothesized reflector are from the shallow marine model of Figure 17. Figure 19 shows that the phase $P d S$ defines an interval of reverberations comprising phases converted from down-going $P$ to up-going $S$ at depths where the vertical gradient in impedance is 
sufficient to generate a phase conversion. The data are consistent with there being a number of significant discontinuities in the shallow subsurface. The shallowest reflector is at about $10 \mathrm{~m}$ beneath the sea floor, there appears to be a sequence of reflectors between about $40 \mathrm{~m}$ and $80 \mathrm{~m}$ depth, and there is also evidence of a reflector at a depth of about $150 \mathrm{~m}$. The amplitude of some of the arrivals in the reverberative interval varies along the record section, which indicates either that the discontinuity is spatially sporadic or that the impedance contrast is variable. Therefore, $P d S$ is a promising phase for imaging shallow discontinuities given an accurate model of $P$ and $S$ velocities above the reflectors. $P d S$ reverberations for shallow reflections are sufficiently complex that their use in practice is not simple, however. Improvements in interpretation will require deconvolving the instrument response and, perhaps, fitting synthetic seismograms to unravel the information in the waveform.

\section{ON THE DESIGN OF MARINE SURVEYS}

To generate seismic wave-fields that exhibit the full complement of phases that provides information about shallow $S$ structures, a marine survey must satisfy certain requirements. We discuss these requirements here.

First and foremost, as Figure 2 shows, the efficiency with which the acoustic wave-field in the sea converts to a shear wave-field in the marine sediments depends strongly on the height of the acoustic source above the sea floor. Moving the source

off the bottom can rapidly attenuate the amplitude of the shear phases and reduce their band-width to very low frequencies. For example, for the marine model shown in Figure 17 moving a source from $1 \mathrm{~m}$ to $10 \mathrm{~m}$ above the sea floor reduces the amplitude of the fundamental mode at $3 \mathrm{~Hz}$ by an order of magnitude and at $5 \mathrm{~Hz}$ by three orders of magnitude. The interface waves produced by sources more than a few meters above the sea floor would, therefore, be limited to very low frequencies, and much of the potential information about shallow shear structures would be missing. 
As examples, Figures 20 and 21 display records generated with acoustic sources about $20 \mathrm{~m}$ and $50 \mathrm{~m}$ above the sea floor, respectively. Only very low frequency $(<3 \mathrm{~Hz})$ fundamental modes and $S$ waves are observed with sources $\sim 20 \mathrm{~m}$ above the sea floor. With sources $\sim 50 \mathrm{~m}$ above the sea floor, no shear arrivals are observed in the raw data, although a very low frequency guided wave is extractable from the record section after the application of an appropriately designed $\omega-k$ filter.

Figures $2 \mathrm{~b}$ and $2 \mathrm{c}$ present two caveats to these observations. First, the excitation of the overtones decays less quickly than the fundamental mode as source height is increased. This means that, as in the Unocal data, guided waves may be observed and interface waves may be missing. As discussed in the previous section, without the interface waves the guided waves are hard to interpret, however. Second, the amplitude decrease and the narrowing of the band width of observation are mitigated if the near surface shear velocities are increased. Thus, regions of active sedimentation, which are characterized by very low near surface shear velocities, will require acoustic sources nearer to the seafloor than regions with more consolidated sediments.

Second, there is a variety of information that is very important in modeling slow low-frequency horizontally propagating waves that may be much less important in studying seismic reflections. For example, complementary to the height-of-source requirement is the requirement that the instrument accurately record low frequency arrivals. Thus, it is desired for the instrument to record down to $1 \mathrm{~Hz}$ and the phase response of the instruments should be known well. The data that we received from Fairfield Industries meet the height-of-source requirement well and the instruments appear to record signals well down to about $2-2.5 \mathrm{~Hz}$, but the phase response of the instruments are unknown to the authors. An instrumental phase advance or lag of, for example, $\pi / 2$ at $3 \mathrm{~Hz}$ would change the measured group velocities by about 1\%. This is lower than the variance in the data by about a factor of $2-3$ and is, therefore, unlikely to affect the 1-D model that we present here appreciably. In a 2-D inversion, however, $1 \%$ errors would become meaningful. The inversion of travel times 
alone makes information about the instrumental amplitude response unnecessary, but if wave-field modeling or $Q$ estimation are desirable, then the amplitude response of the instrument must also be known. Improving the agreement between the observed and simulated wave-fields beyond that exhibited by Figures 5 and 10 will require using this information. Another important piece of information is accurate source and receiver locations. If we desire travel times with an accuracy better than $1 \%$, then we would like source and receiver positions determined to about $1 \mathrm{~m}$ which appears to be beyond current accuracy standards for marine surveys. Finally, if we wish to measure waves propagating at $40 \mathrm{~m} / \mathrm{s}$ to distances of $500 \mathrm{~m}$, say, measured time series must be at least $12 \mathrm{~s}$ in duration. This places a limit on the shot spacing with time.

Third, Love waves are not efficiently generated by acoustic sources in a water layer and, in the three data sets discussed in this paper, we see essentially no sign of them. In land surveys with vibroseis or buried explosive sources, however, they are apparent and may provide useful information complementary to the Rayleigh waves. For example, with observations of both Rayleigh and Love waves one can infer information about transverse isotropy (e.g., Bachman, 1983; Odom et al., 1996). If information from Love waves is desired, sources on towed sleds have been developed by marine geophysicists to generate them (Stoll et al., 1994a) and a few studies have been completed on Love waves in marine sediments (e.g., Bautista and Stoll, 1995). It should be mentioned, however, that Love waves complicate the seismograms such that unambiguous simultaneous determination of Rayleigh and Love wave dispersion may be challenging. Thus the absence of Love waves in marine exploration may be considered a positive feature by some, and more sophisticated sources should only be considered if the added information provided by Love waves is truly needed.

Fourth, as discussed above, the MWI method is ideally suited for a 2-D or 3D inversion. For a multi-dimensional inversion, the sources and receivers should be spaced to match the lateral heterogeneity in the medium. The data set provided to 
us by Fairfield Industries has about a $1 \mathrm{~km}$ receiver spacing and a $25 \mathrm{~m}$ shot spacing. The receiver spacing defines the lateral resolution deep in the model and should, therefore, be no coarser than the minimum lateral resolution that is desired at depth, perhaps $25 \mathrm{~m}$ - $100 \mathrm{~m}$. A shot spacing of $25 \mathrm{~m}$ may be adequate for most purposes. It may be worth noting, however, that the wavelength of the fundamental mode at frequencies above $5 \mathrm{~Hz}$ is $<10 \mathrm{~m}$, so the unambiguous application of any stacking method based on $\omega-k$ analysis would require a shot spacing less than $5 \mathrm{~m}$. This may mean that $\omega-k$ analyses are economically prohibitive.

Fifth, as with any inversion, the quality of the estimated shear velocity model is conditioned by the quality of a priori information used in the inversion. The inversion for shallow shear structure would be improved if information from shallow cores or other sources could be utilized. Particularly useful would be 'ground truth' information on lithological boundaries at which the shear velocity would be expected to jump discontinuously.

\section{CONCLUSIONS}

The seismic wave-field produced by an acoustic source in a water layer overlying marine sediments is rich in information about shallow subsurface shear velocities if the conditions discussed in the previous section are met. It is particularly important for the acoustic source to be very near the sea floor. particularly in regions of active sedimentation. We have argued that multi-component data currently being accumulated as part of offshore exploration can be used to construct reliable models of shear velocities to a depth of about $200 \mathrm{~m}$. Such an $S$ model, together with a $P$ model constructed by inverting refracted $P$-wave travel times, appears to constrain $V_{p}: V_{s}$ well.

The characteristics of the marine seismic wave-field are very site dependent. The observable waves, their travel times, frequency contents, and dispersion properties 
will vary from site to site and the application of MWI must adapt to these variations. In almost any case, however, the waves that will be the most straightforward to interpret are the dispersion characteristics of the fundamental and first overtone interface waves and the travel times of refracted $S$. In the Fairfield Industries data collected off Louisiana, the group and phase velocities of the interface waves provide useful, although not entirely independent, constraints on shear velocities to a depth of 20 $30 \mathrm{~m}$. Although guided waves may be difficult to interpret, they provide valuable information to somewhat greater depths $(\sim 70 \mathrm{~m})$ than the interface waves. The travel times of refracted $S$-waves provide information to the deepest point of penetration of the farthest $S$ waves observed. $S$ is unambiguously observed in the Fairfield Industries data to a source - receiver range of about $1.2 \mathrm{~km}$, which corresponds to an $S$ turning depth of about $225 \mathrm{~m}$.

To date, we have proceeded only part of the way toward exploiting the full richness of the marine seismic wave-field. We have designed the MWI method specifically to allow the incorporation of observations on other types of waves. For example, multiple bounce $S$ phases (e.g., $S S, S S S$ ) are well observed in the Fairfield Industries data and differential travel times between arrivals are relatively easy to measure and interpret to improve constraints at depths between about $30 \mathrm{~m}$ and $100 \mathrm{~m}$. In addition, the converted phase $\operatorname{PdS}$ would be useful to constrain the location of jump discontinuities. Finally, the full capabilities of the MWI method await its application to appropriate data sets for 2-D and 3-D inversions.

\section{ACKNOWLEDGEMENTS}

We are very grateful for permission to use proprietary data granted to us by Fairfield Industries, Unocal, and Western Geophysical. We would like to thank the following individuals for making data available to us: James Gaiser, Neil Jones, and Konstantin Osypov at Western Geophysical; Robert Windels at Fairfield Industries, 
and Gregory Ball at Unocal. In particular, we appreciate the information exchange that we enjoyed with Robert Windels and Gregory Ball and encouragement from Eugene Lavely and Richard Bates at Blackhawk Geometrics, Ken Larner at the Center for Wave Phenomena at Colorado School of Mines, and Jie Zhang of GeoTomo. Fi-

nally, Junseong Heo and Mikhail Barmin assisted in several aspects of the research reported here and Robert Herrmann introduced us to some of the marine geophysics literature.

\section{References:}

Abo-Zena, A.M., 1979, Dispersion function computations for unlimited frequency values, Geophys. J. R. astr. Soc., 58, 91-105.

Akal, T., J.M. Berkson, 1986, eds., Ocean Seismo-Acoustics: Low Frequency Underwater Acoustics, Plenum Press, New York, Proc. SACLANT ASW Research Center Symposium, La Spezia, Italy, June 1985.

Aki, K., and P.G. Richards, 1980, Quantitative Seismology: Theory and Methods, I, W. H. Freeman and Co, San Francisco.

Al-Husseini, M.I., J.B. Glover, and B.J. Barley, 1981, Dispersion patterns of the ground roll in eastern Saudi Arabia, Geophysics, 46, 121-137.

Bachman, R.T., 1983, Elastic anisotropy in marine sedimentary rocks, J. Geophys. Res., 88, 539-545.

Barmin, M.P., A.L. Levshin, and M.H. Ritzwoller, 2000, A fast and reliable method for surface wave tomography, Pure and Appl. Geophys., in press.

Bautista, E. O., R.D. Stoll, 1995, Remote determination of in situ sediment parameters using Love waves, J. Ac. Soc. Am., 98, 1090-1096. 
Bibee, D.L. and L.M. Dorman, 1991, Implications of deep-water seismometer array measurements for Scholte wave propagation, in J.M. Hovem et al. (eds.), Shear Waves in Marine Sediments, Kluwer Academic Publ., Dordrecht, Netherlands, 395-402.

Blonk, B., 1995, Removal of scattered surface waves from seismic data, Thesis Technishe Universiteit Delft.

Caldwell, J., 1999, Marine multi-component seismology, The Leading Edge, 18, no. $11,1274-1282$.

Cara, M., 1973, Filtering of dispersed wave trains, Geophys. J. R. astr. Soc., 33, $65-80,1973$.

Dosso, S. E., and G.H. Brooke, 1995, Measurement of seismo-acoustic ocean-bottom properties in the high Arctic,J. Ac. Soc. Am., 98, 1657-66.

Dziewonski, A.M., Bloch, S., and M. Landisman, 1969. A technique for the analysis of transient seismic signals, Bull. seism. Soc. Am., 59, 427 - 444.

Ernst, F., and G. Herman, 1998, Removal of guided waves from seismic data in laterally varying media, Wave Motion, 28, 173-189.

Essen, H.-H., I. Grevemeyer, R. Herber, and W. Weigel, 1998, Shear-wave velocity in marine sediments on young oceanic crust: constraints from dispersion of Scholte waves, Geophys. J. Int., 132, 227-234.

Ewing, J., A. Carter, G. H. Sutton and N. Barstow, 1992, Shallow Water sediment properties derived from high-frequency shear and interface waves, J. Geophys. Res, 9, 4739-4762.

Frivik, S. A., and J.M. Hovem, 1995, Geotechnical investigation using interface waves for determination of shear properties in the upper seafloor, Challenges of Our 
Changing Global Env., Conf. Proc., OCEANS 95 MTS/IEEE, 1840-5, 1995.

Gabriels, P., R. Snieder, and G. Nolet, 1987, In situ measurements of shear-wave velocity in sediments with higher-mode Rayleigh waves, Geoph. Prosp., 35, 187-196.

Gaiser, J.E., 1996, Multicomponent $V_{p} / V_{s}$ correlation analysis, Geophysics, 61, 11371149 .

Hamilton, E. L., 1979, $V_{p} / V_{s}$ and Poisson's ratios in marine sediments and rocks, $J$. Acoust. Soc. Am., 66 1093-1101.

Hamilton, E.L., 1980, Geoacoustic modeling of the sea floor, J. Acoust. Soc. Am., 68, 1313-1340.

Harvey, D.J., 1981, Seismogram synthesis using normal mode decomposition: the locked mode approximation, Geophys J. R. Astron. Soc., 66, 37-69.

Haskell, N.A., 1953, The dispersion of surface waves on multilayered media, Bull. Seism. Soc. Am., 43, 17-34.

Herrmann, R.B. (Ed.), 1978, Computer programs in earthquake seismology, 2, St. Louis University.

Herrmann, R.B., and D.R. Russell, 1990, Ground roll: Rejection using adaptive phase-matched filters, Geophysics, 55, 776-781.

Hovem, J.M., M.D. Richardson, and R.D. Stoll, 1991, eds., Shear Waves in Marine Sediments, Kluwer Academic Publishers, Dordrecht, 1992, Proc. Conference on Shear Waves in Marine Sediments, La Spezia, Italy, October 1990.

Kawashima, S., M. Kimura, 1998, Determination of shear wave velocity profile using Scholte waves in marine sediments, Jpn. J. Appl. Phys., 1, Regul. Pap., Short Notes Rev. Pap., v.37, 3156-60. 
Kennett, B.L.N., 1983, Seismic wave propagation in stratified media, Cambridge University Press.

Kennett, B. L. N., 1984, Guided-wave propagation in laterally varying media I theoretical development, Geophys. J. R. Astron. Soc.,79, 235-255.

Knopoff, L.,1972, Observation and inversion of surface wave dispersion, Tectonophysics, 13, 497-519.

Levshin, A.L., Pisarenko, V.F., and G.A. Pogrebinsky, 1972, On a frequency-time analysis of oscillations, Ann. Geophys., 28, 211 - 218.

Levshin, A.L., T.B. Yanovskaya, A.V. Lander, B.G. Bukchin, M.P. Barmin, L.I. Ratnikova, and E.N. Its, 1989, Seismic surface waves in a laterally inhomogeneous Earth, (ed. V.I. Keilis-Borok), Kluwer Publ., Dordrecht.

Mari, J.L.,1984, Estimation of static correction for shear-wave profiling using the dispersion properties of Love waves, Geophysics, 49, 1169-1179.

Marsden, D., 1993, Static corrections - A review, Part I, II, and III, The Leading Edge, 12, no. 1-3.

Meissner, R., H. Stumpel, and F. Theilen, 1985, Shear wave studies in shallow sediments, in Handbook of Geophysical Exploration, ed. by G. Dohr, Geophysical Press, London, pp. 224-253.

Neigauz, M.G., and G.V. Shkadinskaya, 1972, Method for calculating surface wave Rayleigh waves in a vertically inhomogeneous half-space, in Computational Seismology (Ed. V.I. Keilis-Borok), Consultants Bureau, New York, 88-92.

Nolet, G., and L.M. Dorman, 1996, Waveform analysis of Scholte modes in ocean sediment layers, Geophys. J. Int., 125, 385-96. 
Odom, R.I., M, Park, J.A. Mercer, R.S. Crosson, and P. Paik, 1996, Effects of transverse isotropy on modes and mode coupling in shallow water, J. Acoust. Soc. Am., 100, 2079-2092.

Park, C.B., R.D. Miller, and J. Xia, 1999, Multichannel analysis of surface waves, Geophysics, 64, 800-808.

Purnell, 1992, Imaging beneath a high-velocity layer using converted waves, Geophysics, 57, 1444-1452.

Rauch, D., 1986, On the role of bottom interface waves in ocean seismoc-acoustics: A review, in Akal, T. and J.M. Berkson (eds.), Ocean Seismo-Acoustics: Low Frequency Underwater Acoustics, 623-641.

Ritzwoller, M.H. and E.M. Lavely, 1995, Three-dimensional seismic models of the Earth's mantle, Revs. of Geophys., 33, 1-66.

Ritzwoller, M.H. and A.L. Levshin, 1998, Eurasian surface wave tomography: Group velocities, J. Geophys. Res., 103, 4839 - 4878.

Rodi, W.L., P. Glover, T.M.C. Li, and S.S. Alexander, 1975, A fast, accurate method for computing group-velocity partial derivatives for Rayleigh and Love modes, Bull. Seis. Soc. Am., 65, 1105-1114.

Saatçilar, R., and N. Canitez, 1988, A method for ground-roll elimination, Geophysics, 53, 894-902.

Scholte, J.G.J., 1958, Rayleigh waves in isotropic and anisotropic elastic media, Meded. en Verhand. KNMI, 72, 9-43.

Schwab, F., and L. Knopoff, 1972, Fast surface wave and free mode computations, in Methods in Computational Physics, 11, 87-180, ed. B.A. Bolt, Academic Press, New York. 
Shieh, C., and R. Herrmann, 1990, Ground roll: rejection using a polarization filter, Geophysics, 55, 1216-1222.

Snieder, R., 1987, Surface wave holography, in Seismic tomography, with applications in global seismology and exploration geophysics, ed. G. Nolet, 323-337, Reidel, Dordrecht.

Smith, D.T., 1986, Geotechnical characteristics of the sea bed related to seismoacoustics, in Akal, T. and J.M. Berkson (eds.), Ocean Seismo-Acoustics: Low Frequency Underwater Acoustics, 483-500.

Stokoe, K.H., and B.L. Rosenblad, 1999, Offshore geotechnical investigations with shear waves, 1999 Offshore Technology Conference, Houston, Texas, 3-6 May, OTC 10823, 1-9.

Stoll, R.D., 1989, Sediment Acoustics, Springer - Verlag, New York.

Stoll, R.D., G.M. Bryan, R. Flood, D. Chayes, and P. Manley, 1988, Shallow seismic experiments using shear waves, J. Acoust. Soc. Am., 83, 93-102.

Stoll, R.D., E. Bautista, and R. Flood, New tools for studying sea-floor geotechnical and geoacoustic properties, J. Acoust. Soc. Am., 96, 2937-2944, 1994a.

Stoll, R.D., G.M. Bryan, and E. Bautista, 1994b, Measuring lateral variability of sediment geoacoustic properties, J. Acoust. Soc. Am, 96, 427-438.

Vdovin, O., J.A. Rial, A.L. Levshin, and M.H. Ritzwoller, 1999, Group-velocity tomography of South America and the surrounding oceans, Geophys. J. Int., 136, 324-340.

Woodward, R.L. and G. Masters, 1991, Upper mantle structure from long-period differential travel times and free oscillation data, Geophys. J. Int., 109, 275293. 
Xia, J., R. Miller, and C. Park, 1999, Estimation of shear velocity by inverting Rayleigh wave phase velocity, Geophysics, 64, 691-700.

Zhu, X., Altan S., and J. Li, 1999, Recent advances in multi-component processing, The Leading Edge, 18, no. 11, 1283-1288.

\section{Appendix. Normal Mode Synthetic Seismograms for a Vertically Inhomoge- neous Medium Underlying a Fluid Layer}

We consider surface wave propagation in a laterally homogeneous isotropic elastic half-space in which the upper layer is a fluid. We use a source-centered cylindrical coordinate system $(r, \varphi, z), 0 \leq r<\infty, 0 \leq \varphi<2 \pi, 0 \leq z<\infty$. The 1D isotropic model is characterized by a piece-wise continuous vector-function $\mathbf{m}(z)$, $\mathbf{m}=(\alpha, \beta, \rho)$, where $\alpha$ and $\beta$ are $P$ - and $S$-velocities and $\rho$ is density. We assume that there exists a depth $Z$ at which $\alpha$ and $\beta$ reach their maximum values and are constant at depths $z \geq Z$.

Let a point explosive source situated at the point $(0,0, h)$ and be described by the seismic moment tensor $\mathbf{M} H(t)$, where $t$ is time, and $\mathbf{M}=M_{0} \mathbf{I}$ where $\mathbf{I}$ is the identity matrix. Such a source does not generate $\mathrm{SH}$ and Love waves. $H(t) \approx 0$ if $t<0$, but if $t \geq 0$ then $H(t)=\exp \left(-t / t_{0}\right)$. The characteristic time of the explosion, $t_{o}$, is a small constant which is a function of the source mechanism. The Fourier transform of $H(t)$ is defined as

$$
\mathcal{H}(\omega)=\int_{0}^{\infty} H(t) \exp (-i \omega t) d t
$$

The receiver is located at the point $(r, \varphi, d)$ on the surface of the solid medium, where $d$ is the thickness of the liquid layer.

A synthetic seismogram of the displacement observed at the receiver is

$$
\mathbf{u}(t, r, d)=\frac{1}{2 \pi} \int_{-\infty}^{\infty} \mathbf{D}(\omega, r, h) \exp (i \omega t) d \omega
$$


where $r$ is the epicentral distance, and $h$ is the source depth. Due to the axial symmetry of the problem relative to the vertical axis there is no dependence of the seismogram on the epicentral azimuth $\varphi$.

The displacement spectrum can be presented as a sum of two terms

$$
\mathbf{D}=\mathbf{D}_{\mathbf{L}}+\mathbf{D}_{\mathrm{NM}}
$$

The term $\mathbf{D}_{\mathbf{N M}}$ describes the contribution of body waves, guided and interface waves of Rayleigh type which propagate with phase velocities (inverse slownesses) less than $\beta(Z)$, the maximum shear velocity in the medium. In particular, it includes the contribution of refracted (diving) and head S-waves in which rays are confined to the depths $0 \leq z<Z$. The energy of these waves does not leak into the underlying half-space $(z>Z)$. The term $\mathbf{D}_{\mathbf{L}}$ in equation $(\mathrm{A} 4)$ describes waves that penetrate or leak into the half-space, mostly $\mathrm{P}$ waves and subcritical S and PdS reflections. Their phase velocities are higher than $\beta(Z)$. Here we consider only the contribution of waves representable by normal modes. The set of waves represented by $\mathbf{D}_{\mathbf{L}}$ can be minimized by making $\beta(Z)$ and $Z$ very large, which is the basic idea of the locked mode formalism of Harvey (1981).

The normal mode spectrum may be further decomposed into fundamental and overtone modes depending on the vertical order $n$ of the mode, $\mathbf{D}_{\mathbf{N M}}=\sum_{n} \mathbf{D}_{\mathbf{N M}}{ }^{(n)}$, where the fundamental surface wave has $n=0$. Hereinafter we suppress the index $n$. Let $\mathbf{e}_{r}, \mathbf{e}_{z}$ be the local unit vectors at the receiver, so $\mathbf{D}_{\mathbf{N M}}=D_{r} \mathbf{e}_{r}+D_{z} \mathbf{e}_{z}$, where $r$-direction is radially outward from the source along the straight line linking source to receiver.

With these definitions the complex displacement spectrum of the fundamental surface waves may be asymptotically presented as (Aki \& Richards, 1980; Levshin et al., 1989)

$$
D_{R z}(\omega, r, h)=\frac{\exp (-i \pi / 4)}{\sqrt{8 \pi}} \frac{\exp \left(-i k_{R}(\omega) r\right)}{\sqrt{k_{R}(\omega) r}}
$$




$$
\begin{gathered}
\times \mathcal{A}_{R}(\omega, h) \mathcal{H}(\omega), \\
D_{R r}(\omega, r, h)=\exp (-i \pi / 2) D_{R z}(\omega, r, h) \epsilon(\omega),
\end{gathered}
$$

if $k_{R} r>>2 \pi, r>>h$. Here $k_{R}(\omega)$ is a horizontal wavenumber, $\mathcal{C}_{R}(\omega)=\omega / k_{R}$ is a phase velocity, $\mathcal{U}_{R}(\omega)=\left(d k_{R} / d \omega\right)^{-1}$ is a group velocity, and $\epsilon(\omega)$ is the eccentricity of the particle motion evaluated at the ocean bottom $(z=d)$. The second term in equation (A5) describes propagation effects and the third term characterizes the excitation of a given mode by the source. We define excitation spectrum as:

$$
\mathcal{A}_{R}(\omega, h)=\frac{\left[U^{\prime}(\omega, h)+k_{R}(\omega) V(\omega, h)\right] M_{0}}{\mathcal{C}_{R}(\omega) \mathcal{U}_{R}(\omega) I_{R}(\omega)} .
$$

The values of $k_{R}(\omega)$ are found as the eigenvalues of a one-dimensional boundaryvalue problem for Rayleigh waves described, e.g., by Aki \& Richards (1980) or by Levshin et al. (1989). $U(\omega, z)$ and $V(\omega, z)$ are the vertical and horizontal components of the vector-eigenfunction of this problem. The normalization integral $I_{R}$ is proportional to the kinetic energy of the given Rayleigh mode and is equal to $\int_{0}^{\infty} \rho(z)\left[U^{2}(\omega, z)+V^{2}(\omega, z)\right] d z$. There are numerous algorithms and codes dedicated to numerical solutions of this boundary-value problem (Haskell, 1953; Neigauz \& Shkadinskaya, 1972; Schwab \& Knopoff, 1972; Herrmann, 1978, Abo-Zena, 1979, Kennett, 1983).

To introduce attenuation to the elastic model we add to the vector-function $\mathbf{m}(z)$ an extra component $Q_{S}(z, \omega)$ which characterizes the attenuation of shear waves as a function of depth and frequency. This is done by adding to the elastic moduli small complex perturbations corresponding to the given model of $Q_{S}(z, \omega)$ (Aki \& Richards, 1980; Levshin et al., 1989). Here, we assume that $Q_{S}$ is frequency-independent, and the relation between attenuation of $\mathrm{P}$ - and S-waves is $Q_{P}(z) / Q_{S}(z)=4 \beta^{2}(z) / 3 \alpha^{2}(z)$. Then, in addition to the functions describing surface wave spectra mentioned above we are able to calculate the apparent surface wave quality factor $Q_{R}^{(n)}(\omega)$ for each normal mode. 
In summary, we calculate synthetic seismograms for vertical and radial (inline) displacements generated by the explosive source for Rayleigh waves by means of the following equations:

$$
\begin{aligned}
& u_{R z}(t, r, d)= \\
& \frac{1}{2 \pi} \int_{-\infty}^{\infty} \Phi(\omega) \sum_{n=0}^{N} D_{R z}^{(n)}(\omega, r, h) \exp (i \omega t) d \omega, \\
& u_{R r}(t, r, d)= \\
& \frac{1}{2 \pi} \int_{-\infty}^{\infty} \Phi(\omega) \sum_{n=0}^{N} D_{R r}^{(n)}(\omega, r, h) \exp (i \omega t) d \omega .
\end{aligned}
$$

where

$$
\begin{aligned}
D_{R z}^{(n)}= & \frac{\exp (-i \pi / 4)}{\sqrt{8 \pi}} \mathcal{A}_{R}^{(n)}(\omega, h) \mathcal{H}(\omega) \\
& \times \frac{\exp \left(-i k_{R}^{(n)}(\omega) r-\omega r /\left(2 Q_{R}^{(n)}(\omega) U_{R}^{(n)}(\omega)\right)\right)}{\sqrt{k_{R}^{(n)}(\omega) r}} \\
D_{R r}^{(n)}= & D_{R z}^{(n)} \epsilon^{(n)}(\omega) \exp (-i \pi / 2)
\end{aligned}
$$

with $\mathcal{A}_{R}^{(n)}$ defined by (A8) and $\mathcal{H}$ defined by (A1) and (A2). $\Phi(\omega)$ describes the bandpass filter simulating the instrument response, and $N$ is the number of the highest mode existing for the frequency range defined by this filter. 


\section{FIGURES}

FIG. 1. Separate modal contributions to the horizontal component (COME) of the synthetic waveform FF0013 in the record section found in Fig. 5. The complete synthetic seismogram is at the top, labeled as 'sum' left of the trace. The fundamental mode and the first ten overtones that together sum to the complete synthetic waveform are labeled with the mode number and arrayed below it. Amplitude normalization factors are arrayed right of every trace. The approximate location of the $S$ wave, the first and second guided waves $\left(G_{1}, G_{2}\right)$, and the fundamental interface wave $\left(I_{0}\right)$ are indicated on the sum. The guided wave $G_{1}$ is mostly the sum of first and second overtones, $G_{2}$ is composed of the third and fourth overtones, and the $S$ wave is the sum of these and higher modes. The record is at a range of $400 \mathrm{~m}$ from the source.

FIG. 2. Excitation curves $(A(\omega)$ from the Appendix) for shots in the water at different elevations above the sea floor with receivers on the sea floor. (a) The fundamental mode with the shallow marine model in Fig. 17. (b) The fundamental mode with a model in which the shear velocities in the shallow marine model are multiplied everywhere by two. (c) The fourth overtone with the shallow marine model. Elevations above the sea floor are indicated next to the associated excitation function. The product of these curves with the displacement eigenfunctions (e.g., Fig. 4) gives the amplitude of the response of the medium to a point source in the fluid layer. No attenuation is included.

FIG. 3. Dispersion curves for the shallow marine model shown in Fig. 17. (a) Group velocity curves. (b) Phase velocity curves. Mode numbers are labeled on the plot from the fundamental mode (0) up to the eleventh overtone (11).

FIG. 4. Displacement eigenfunctions computed from the marine model in Figure 17 for (a) the fundamental mode $\left(I_{0}\right)$, (b) the first overtone $\left(I_{1}\right)$, and (c) the guided wave $\left(G_{1}\right)$. Vertical and horizontal eigenfunctions are plotted as solid and dashed lines, respectively. The frequency is indicated next to each eigenfunction: $2,3,5,10 \mathrm{~Hz}$ for the fundamental, $3,5,7 \mathrm{~Hz}$ for the first overtone, and 
$3,4,8 \mathrm{~Hz}$ for the guided wave. The guided wave is a mixture of modes and what is shown in (c) are the eigenfunctions for the first overtone at $3 \mathrm{~Hz}$, the second overtone at $4 \mathrm{~Hz}$, and the third overtone at $8 \mathrm{~Hz}$. The eigenfunctions are unitless and are normalized such that the vertical eigenfunctions are unity at the solid-water interface ( 0 depth) and the associated horizontal eigenfunction has the same normalization. Thus, for each mode at each frequency the ratio of horizontal to vertical eigenfunction is as presented in this figure, but amplitudes are not comparable across mode types and frequencies. The scales for the horizontal eigenfunctions for the fundamental mode at $2 \mathrm{~Hz}$ and the guided waves have been divided by 2 and 2.5, respectively, so that the vertical eigenfunction would be visible. The vertical grey lines indicate the zero levels.

FIG. 5. Vertical (top) and horizontal (bottom) component synthetic seismograms computed by normal mode summation using the fundamental mode and the first 11 overtones. The basis model is the marine model presented in the inversion section of the paper (Fig. 17). Receivers are placed at $25 \mathrm{~m}$ intervals ranging from $100 \mathrm{~m}$ to $1200 \mathrm{~m}$ from the source. The main wave types are indicated with dashed lines: phases $I_{0}, I_{1}, G_{1}$, and $S$ are described in the text and $S S$ and $S S S$ are double and triple surface bounce phases, respectively. No instrument responses are applied, and ground motion is displacement. The horizontal component is the direction radially away from the source at the receiver. The seismograms have been band pass filtered with corners at 1.5 and $8 \mathrm{~Hz}$.

FIG. 6. Frequency-time diagrams constructed from the synthetic seismogram FF0013 in Figs. 1 and 5. Darker shades denote larger amplitudes. (Left) Vertical component (COMZ). (Right) Horizontal component (COME). These plots are sometimes called Gabor diagrams. The records are 400 $\mathrm{m}$ from the source. Theoretical group velocity curves from Fig. 3a are overplotted and wave types are indicated. The region of the diagram that contributes to the guided wave $G_{1}$ is circled and $G_{2}$ is also indicated.

FIG. 7. Turning depths for the refracted phases $S$ and $P$ as well as the bounce phases $S S$ and $S S S$ in the shallow marine model (Fig. 17). Because the model is parameterized as a stack of 
constant velocity layers, these curves are largely piece-wise constant indicating head waves below a jump discontinuity in the model. The $S$ phases are solid lines and $P$ is a dashed line.

FIG. 8. Theoretical PdS travel times computed from the shallow marine model in Fig. 17. The solid lines indicate the theoretical curves for reflectors ranging in depth from $10 \mathrm{~m}$ to $250 \mathrm{~m}$ indicated to the right of each curve and define a "reverberative interval" in which $P d S$ arrives. The dashed lines are the travel time curves for single refracted $P$ and $S$. ( $P d S$ is a converted phase in which a downward $P$ wave is partially converted to an upward $S$ wave at a reflector at a depth of $d$ meters.)

FIG. 9. Layout of the shots and receivers for the marine data provided by Fairfield Industries. Each shot is denoted by a star and each receiver by a diamond. There are three receiver gathers divided into 'northern' and 'southern' parts so that, for example, $1 \mathrm{~N}$ denotes the northern half of shots for the first receiver. (The actual geographical directions are unknown to the authors.)

FIG. 10. Marine data from Fairfield Industries. Water depth is about $5 \mathrm{~m}$ and acoustic sources are about $3 \mathrm{~m}$ above the sea floor. Vertical (top) and horizontal (bottom) component receiver gathers are from shot line $1 \mathrm{~N}$ for the vertical component and shot line $2 \mathrm{~S}$ for the horizontal component (see Fig. 9). Dashed lines on the vertical records are approximate arrival times of the fundamental Rayleigh wave $\left(I_{0}\right)$ and the first guided wave $\left(G_{1}\right)$ and on the horizontal records they are the refracted shear phase $S$, the refacted surface bounce phases $S S$ and $S S S$, and the group arrival times of the first Rayleigh overtones $\left(I_{1}\right)$ and two guided waves $\left(G_{1}, G_{2}\right)$. Shots range from about $50 \mathrm{~m}$ to about $1.2 \mathrm{~km}$ from the associated receiver. The vertical and horizontal records are low pass filtered with a high frequency corner at $5 \mathrm{~Hz}$ and $8 \mathrm{~Hz}$, respectively, to accentuate these low frequency shear phases.

FIG. 11. Example of frequency-time diagrams for the Fairfield Industries data: record FF230 (Fig. 10) is from receiver gather $2 \mathrm{~S}$ (Fig. 9). The vertical component is at left and a horizontal component at right. Theoretical group velocity curves (Fig. 3a) computed from the shallow marine 
model (Fig. 17) are overplotted. Wave types are indicated. The shot-receiver distance is about 500 m.

FIG. 12. Examples of the measurement of $\mathrm{S}$ velocities for a set of horizontal component records on the $1 \mathrm{~N}$ shot line (Fig. 9) from the marine Fairfield Industries data. A low pass filter with high frequency corner at $8 \mathrm{~Hz}$ was applied to reduce the high frequency noise (multiple $\mathrm{P}$ arrivals and $\mathrm{P}$ to $\mathrm{S}$ conversions) preceding the S arrivals. Shot - receiver distances range from $341 \mathrm{~m}$ to $541 \mathrm{~m}$.

FIG. 13. Location of the wave paths of the dispersion measurements obtained from the Fairfield Industries data: (a) fundamental Rayleigh wave $\left(I_{0}\right)$ group velocity, (b) fundamental Rayleigh wave phase velocity, and (c) first overtone Rayleigh wave $\left(I_{1}\right)$ group velocity. The vertical scales are identical to that in Fig. 9 and the horizontal scales indicate the measurement number. The wave paths of 115, 270 and 148 separate measurements are shown in panels (a) - (c), respectively.

FIG. 14. Average of interface and guided wave dispersion and $S$ wave travel time measurements obtained from the Fairfield Industries data. (Left) Fundamental mode $\left(U_{0}\right)$, first overtone $\left(U_{1}\right)$, and guided wave $\left(G_{1}, G_{2}\right)$ group velocities and the phase velocity of the fundamental $\left(C_{0}\right)$ are shown. (Right) Average $S$ wave travel time.

FIG. 15. Relative rms misfit between the measurements and the mean values shown in Fig. 14 for (a) the fundamental mode Rayleigh wave group velocity $\left(I_{0}\right)$, (b) the first overtone Rayleigh wave group velocity $\left(I_{1}\right),(\mathrm{c})$ the fundamental mode Rayleigh wave phase velocity $\left(C_{0}\right)$, and (d) the $S$ wave travel time. When the group velocities from Fig. 16 are taken as the reference, the misfit to the fundamental Rayleigh wave group velocity measurements reduces to the dashed line in (a). Interface wave results are plotted versus frequency and the $S$ wave results versus horizontal distance. All units are percent.

FIG. 16. Spatial variation of group velocity dispersion for the fundamental Rayleigh wave $\left(I_{0}\right)$ 
from the marine Fairfield Industries data. Distances refer to the spatial scale in Fig. 9.

FIG. 17. One of the shallow marine models that fit the average composite data set shown in Fig. 14: (a) shear velocity $V_{s}$, (b) compressional velocity $V_{p}$, and (c) $V_{p} / V_{s}$. This is the model used throughout this paper.

FIG. 18. Results of the Monte-Carlo inversion for $V_{s}$. (a) The best fitting model and $2 \sigma$ error bars, where $\sigma$ is the standard deviation in each layer of the ensemble of acceptable models. The best fitting model is shown as the solid line. (b) Vertical shear wave travel time to the surface computed from each depth and $2 \sigma$ error bars, where $\sigma$ is the standard deviation of the travel time for the ensemble of acceptable models.

FIG. 19. Theoretical arrival times of $P$ and the converted phase $P d S$ are shown as dashed lines plotted over horizontal waveforms from the $2 \mathrm{~N}$ receiver gather (Fig. 9). The arrivals are computed with the shallow marine model shown in Fig. 17. Arrivals from different hypothesized reflectors are labeled with the depth of the reflector at the top of the figure. The amplitudes of the waveforms are modified by a geometrical spreading factor to facilitate comparison.

FIG. 20. Marine data from Western Geophysical. Water depth is slightly greater than $20 \mathrm{~m}$ on average and acoustic sources are about $20 \mathrm{~m}$ above the ocean bottom. Dashed lines represent approximate arrival times of $I_{0}, I_{1}$, and $S$. Shot WS2235 is about $32 \mathrm{~m}$ from the receiver and shot WS2275 is about $1.025 \mathrm{~km}$. All records have been low pass filtered with a high frequency corner at $3 \mathrm{~Hz}$. All records are horizontal components.

FIG. 21. Marine data from Unocal: (a) unfiltered, (b) low pass frequency filtered (high frequency corner at $3 \mathrm{~Hz}$ ), and (c) frequency-wavenumber filtered. Water depth is about $50 \mathrm{~m}$ and the acoustic source is about the same height above the ocean bottom. Refracted shear waves, guided waves, and interface waves are not seen on the raw data, but very low frequency shingled guided waves are 
barely visible on low pass filtered record sections. The guided waves, probably from the first overtone, are more clearly seen on records that have been filtered in the frequency-wavenumber domain. Shot spacing is about $25 \mathrm{~m}$ and source-receiver distances here range from $\sim 0$ to $\sim 750 \mathrm{~m}$. 


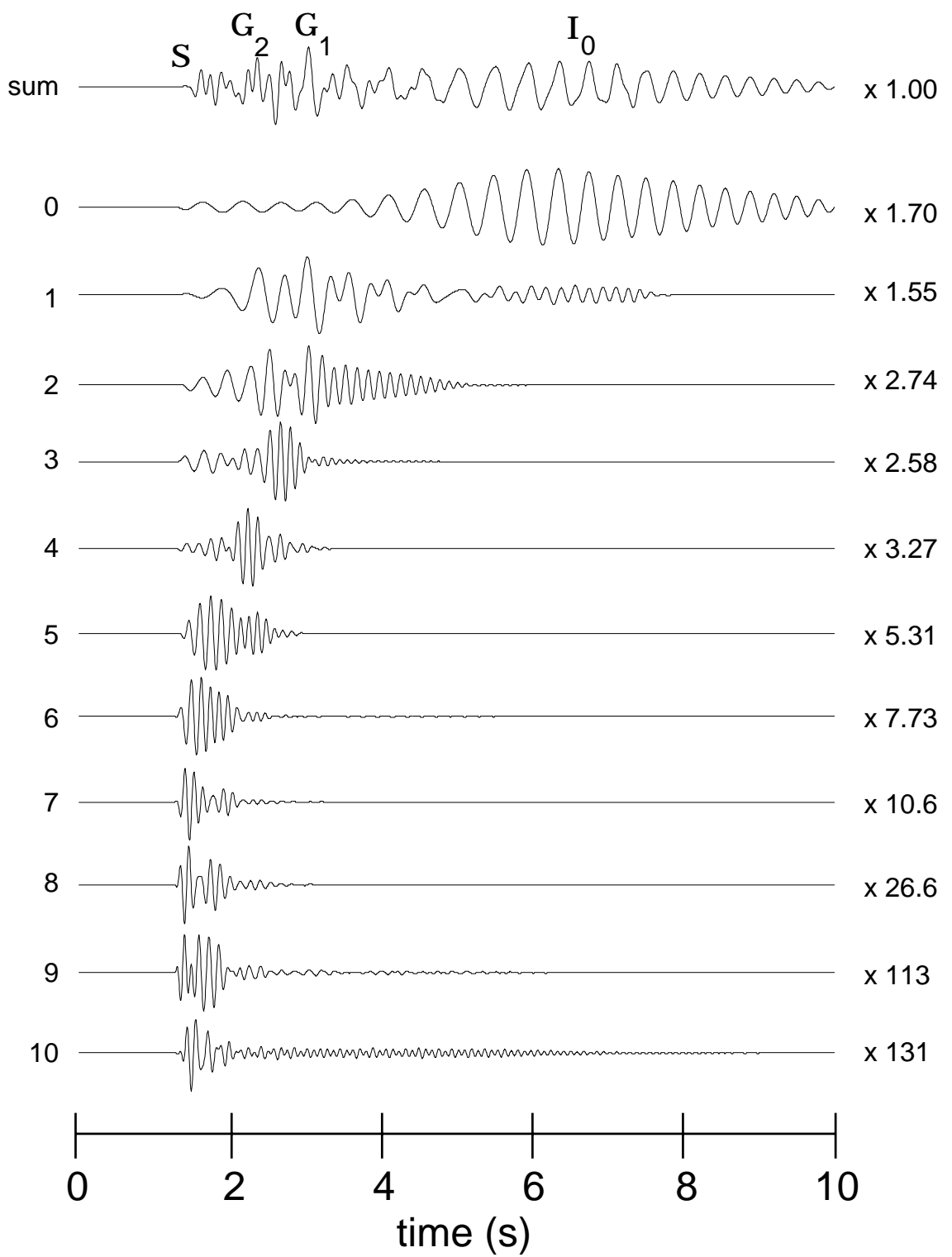

FIG. 1. Separate modal contributions to the horizontal component (COME) of the synthetic waveform FF0013 in the record section found in Fig. 5. The complete synthetic seismogram is at the top, labeled as 'sum' left of the trace. The fundamental mode and the first ten overtones that together sum to the complete synthetic waveform are labeled with the mode number and arrayed below it. Amplitude normalization factors are arrayed right of every trace. The approximate location of the $S$ wave, the first and second guided waves $\left(G_{1}, G_{2}\right)$, and the fundamental interface wave $\left(I_{0}\right)$ are indicated on the sum. The guided wave $G_{1}$ is mostly the sum of first and second overtones, $G_{2}$ is composed of the third and fourth overtones, and the $S$ wave is the sum of these and higher modes. The record is at a range of $400 \mathrm{~m}$ from the source. 
(a)

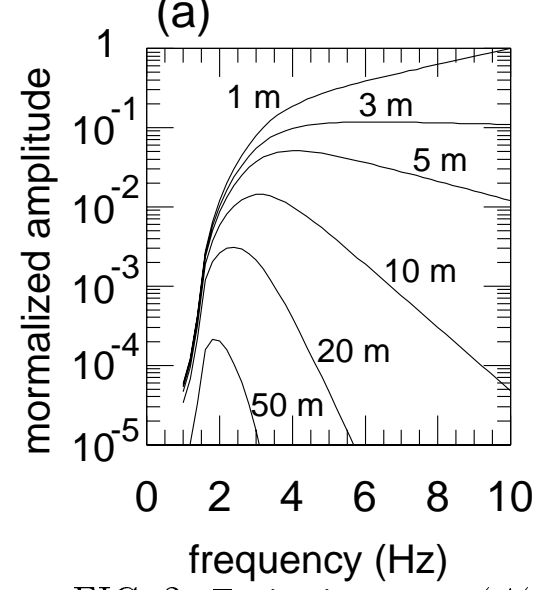

(b)

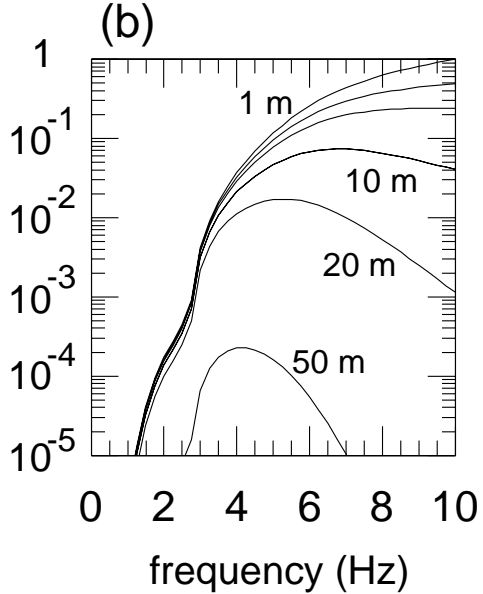

(c)

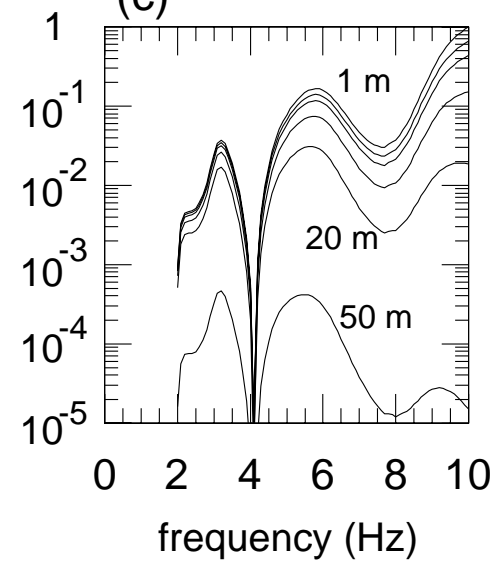

FIG. 2. Excitation curves $(A(\omega)$ from the Appendix) for shots in the water at different elevations above the sea floor with receivers on the sea floor. (a) The fundamental mode with the shallow marine model in Fig. 17. (b) The fundamental mode with a model in which the shear velocities in the shallow marine model are multiplied everywhere by two. (c) The fourth overtone with the shallow marine model. Elevations above the sea floor are indicated next to the associated excitation function. The product of these curves with the displacement eigenfunctions (e.g., Fig. 4) gives the amplitude of the response of the medium to a point source in the fluid layer. No attenuation is included. 

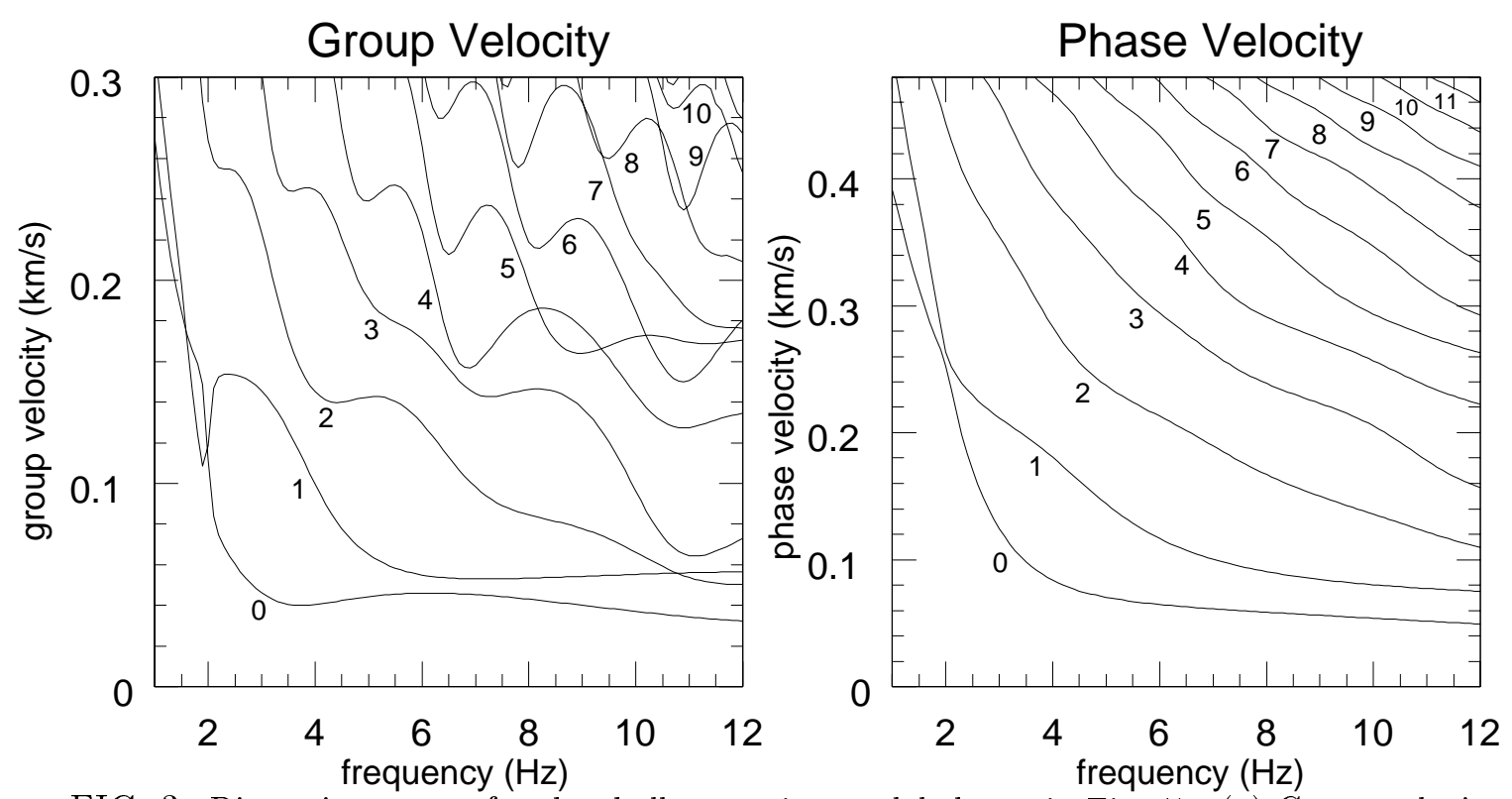

FIG. 3. Dispersion curves for the shallow marine model shown in Fig. 17. (a) Group velocity curves. (b) Phase velocity curves. Mode numbers are labeled on the plot from the fundamental mode (0) up to the eleventh overtone (11). 

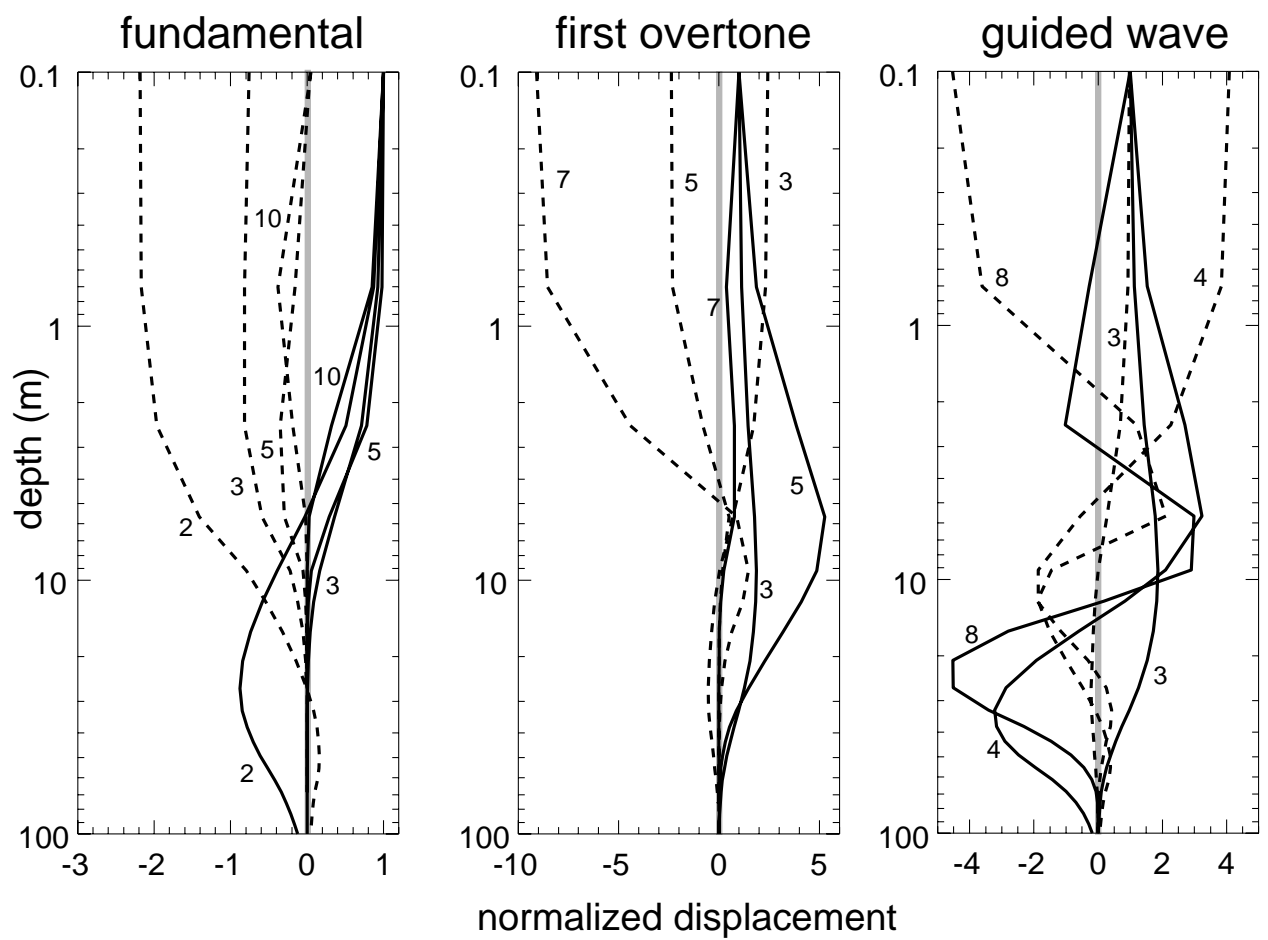

FIG. 4. Displacement eigenfunctions computed from the marine model in Figure 17 for (a) the fundamental mode $\left(I_{0}\right)$, (b) the first overtone $\left(I_{1}\right)$, and (c) the guided wave $\left(G_{1}\right)$. Vertical and horizontal eigenfunctions are plotted as solid and dashed lines, respectively. The frequency is indicated next to each eigenfunction: $2,3,5,10 \mathrm{~Hz}$ for the fundamental, $3,5,7 \mathrm{~Hz}$ for the first overtone, and $3,4,8 \mathrm{~Hz}$ for the guided wave. The guided wave is a mixture of modes and what is shown in (c) are the eigenfunctions for the first overtone at $3 \mathrm{~Hz}$, the second overtone at $4 \mathrm{~Hz}$, and the third overtone at $8 \mathrm{~Hz}$. The eigenfunctions are unitless and are normalized such that the vertical eigenfunctions are unity at the solid-water interface ( 0 depth) and the associated horizontal eigenfunction has the same normalization. Thus, for each mode at each frequency the ratio of horizontal to vertical eigenfunction is as presented in this figure, but amplitudes are not comparable across mode types and frequencies. The scales for the horizontal eigenfunctions for the fundamental mode at $2 \mathrm{~Hz}$ and the guided waves have been divided by 2 and 2.5 , respectively, so that the vertical eigenfunction would be visible. The vertical grey lines indicate the zero levels. 

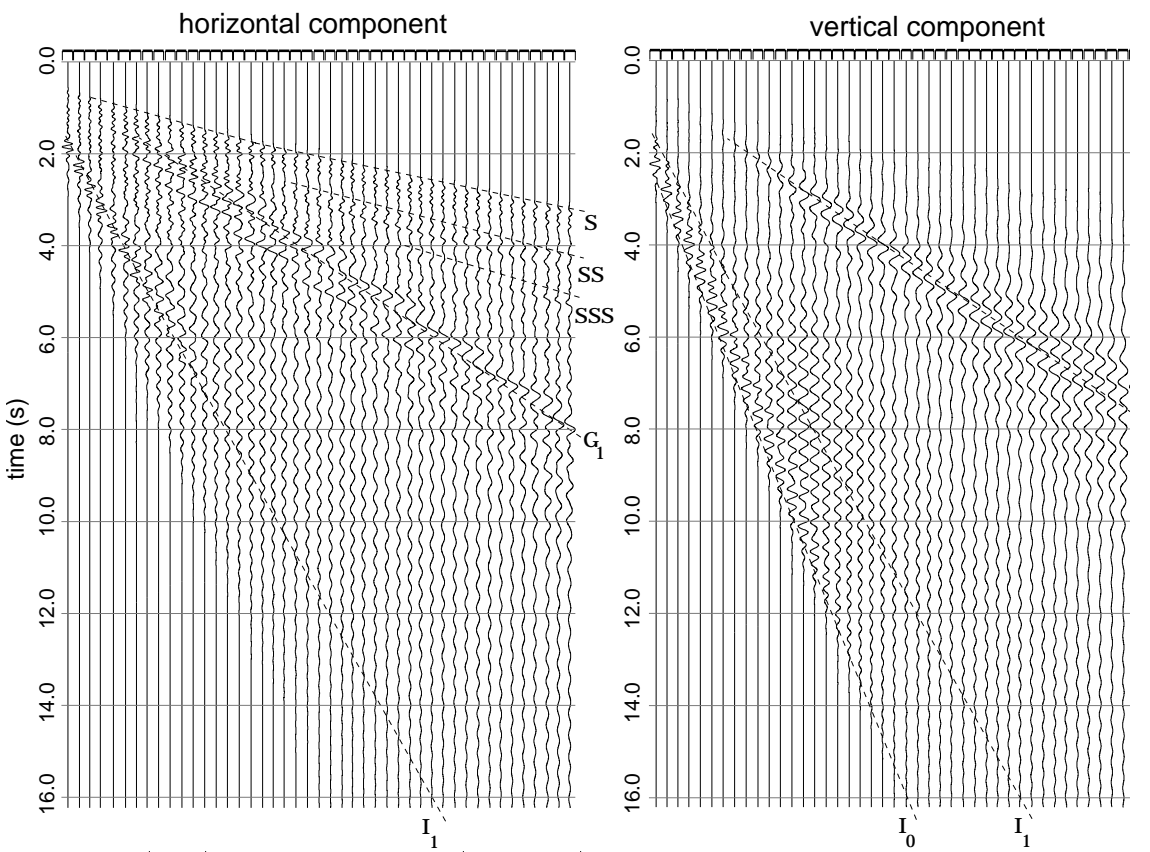

FIG. 5. Vertical (top) and horizontal (bottom) component synthetic seismograms computed by normal mode summation using the fundamental mode and the first 11 overtones. The basis model is the marine model presented in the inversion section of the paper (Fig. 17). Receivers are placed at $25 \mathrm{~m}$ intervals ranging from $100 \mathrm{~m}$ to $1200 \mathrm{~m}$ from the source. The main wave types are indicated with dashed lines: phases $I_{0}, I_{1}, G_{1}$, and $S$ are described in the text and $S S$ and $S S S$ are double and triple surface bounce phases, respectively. No instrument responses are applied, and ground motion is displacement. The horizontal component is the direction radially away from the source at the receiver. The seismograms have been band pass filtered with corners at 1.5 and $8 \mathrm{~Hz}$. 

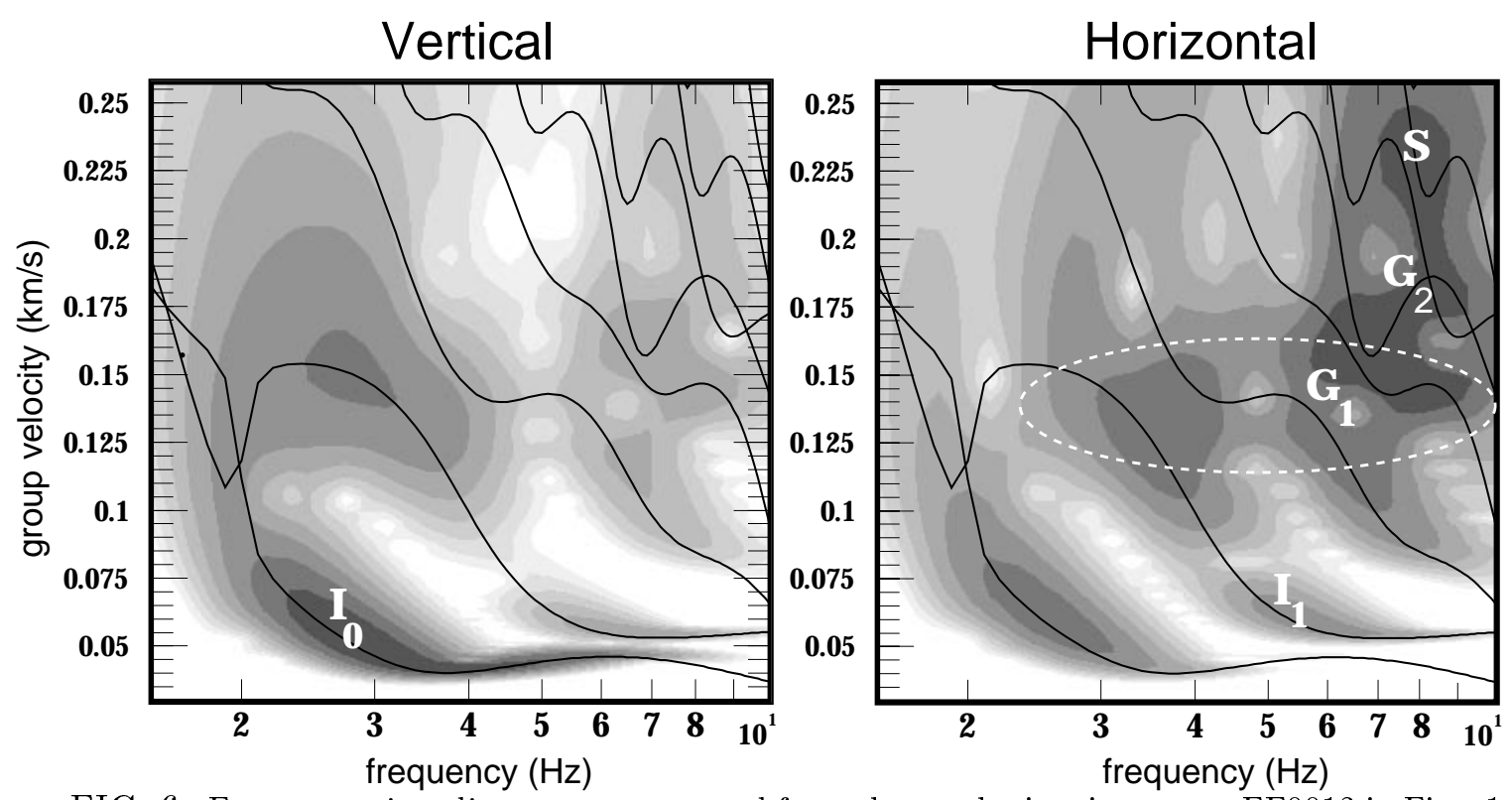

FIG. 6. Frequency-time diagrams constructed from the synthetic seismogram FF0013 in Figs. 1

and 5. Darker shades denote larger amplitudes. (Left) Vertical component (COMZ). (Right) Horizontal component (COME). These plots are sometimes called Gabor diagrams. The records are 400 $\mathrm{m}$ from the source. Theoretical group velocity curves from Fig. 3a are overplotted and wave types are indicated. The region of the diagram that contributes to the guided wave $G_{1}$ is circled and $G_{2}$ is also indicated. 


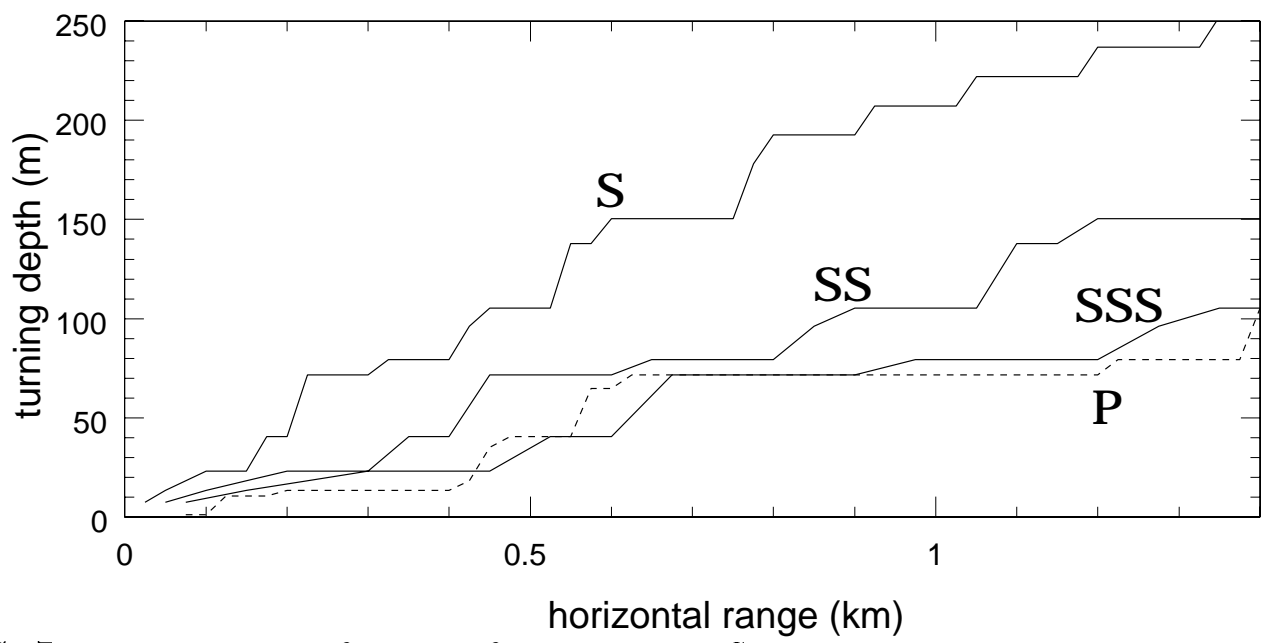

FIG. 7. Turning depths for the refracted phases $S$ and $P$ as well as the bounce phases $S S$ and $S S S$ in the shallow marine model (Fig. 17). Because the model is parameterized as a stack of constant velocity layers, these curves are largely piece-wise constant indicating head waves below a jump discontinuity in the model. The $S$ phases are solid lines and $P$ is a dashed line. 


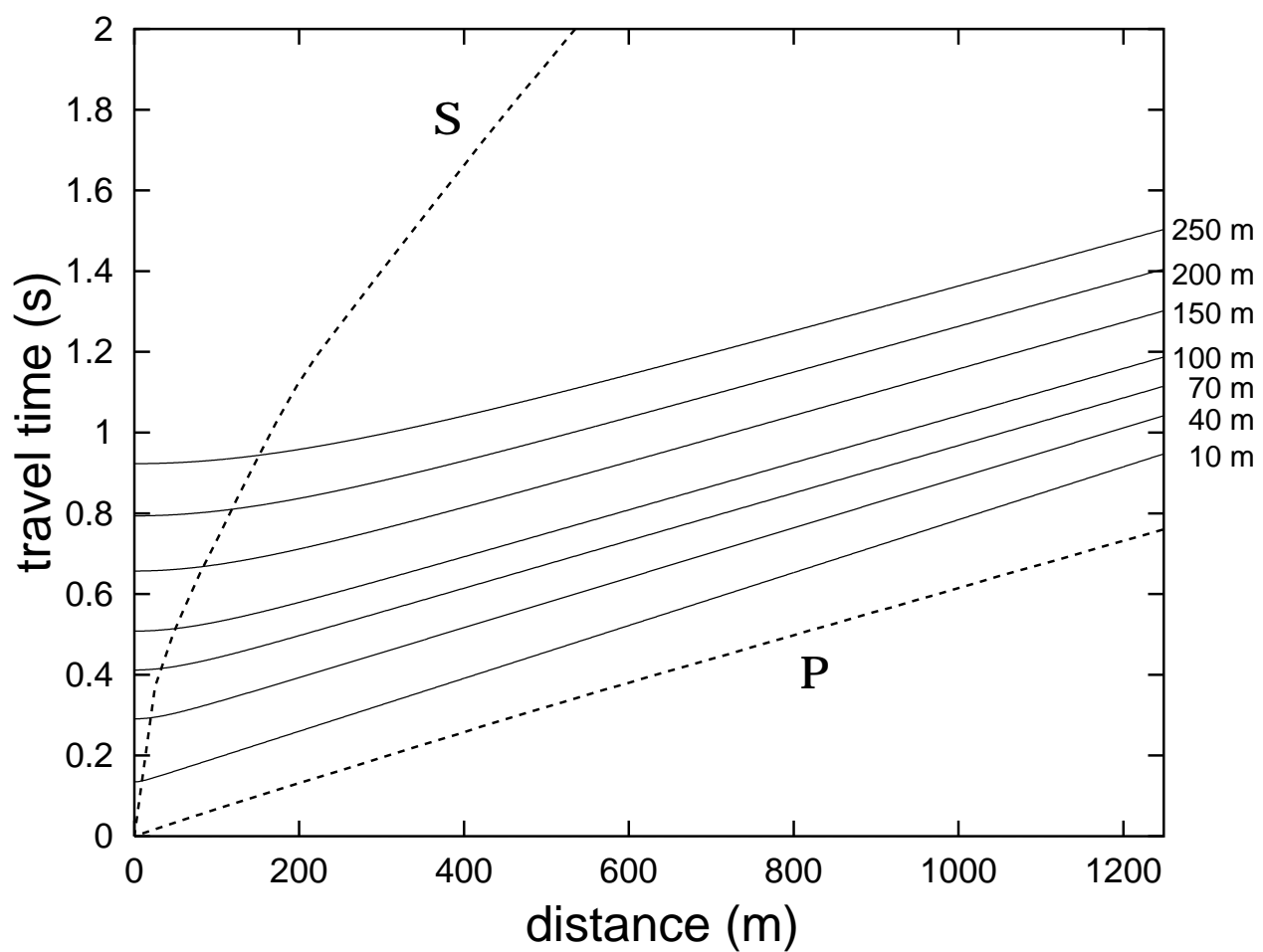

FIG. 8. Theoretical $P d S$ travel times computed from the shallow marine model in Fig. 17. The solid lines indicate the theoretical curves for reflectors ranging in depth from $10 \mathrm{~m}$ to $250 \mathrm{~m}$ indicated to the right of each curve and define a "reverberative interval" in which $P d S$ arrives. The dashed lines are the travel time curves for single refracted $P$ and $S$. ( $P d S$ is a converted phase in which a downward $P$ wave is partially converted to an upward $S$ wave at a reflector at a depth of $d$ meters.) 


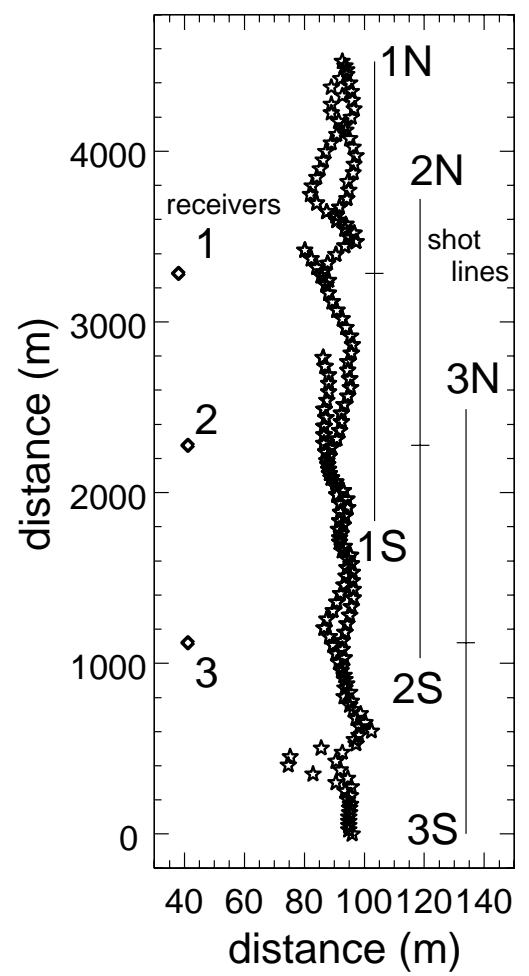

FIG. 9. Layout of the shots and receivers for the marine data provided by Fairfield Industries.

Each shot is denoted by a star and each receiver by a diamond. There are three receiver gathers divided into 'northern' and 'southern' parts so that, for example, $1 \mathrm{~N}$ denotes the northern half of shots for the first receiver. (The actual geographical directions are unknown to the authors.) 


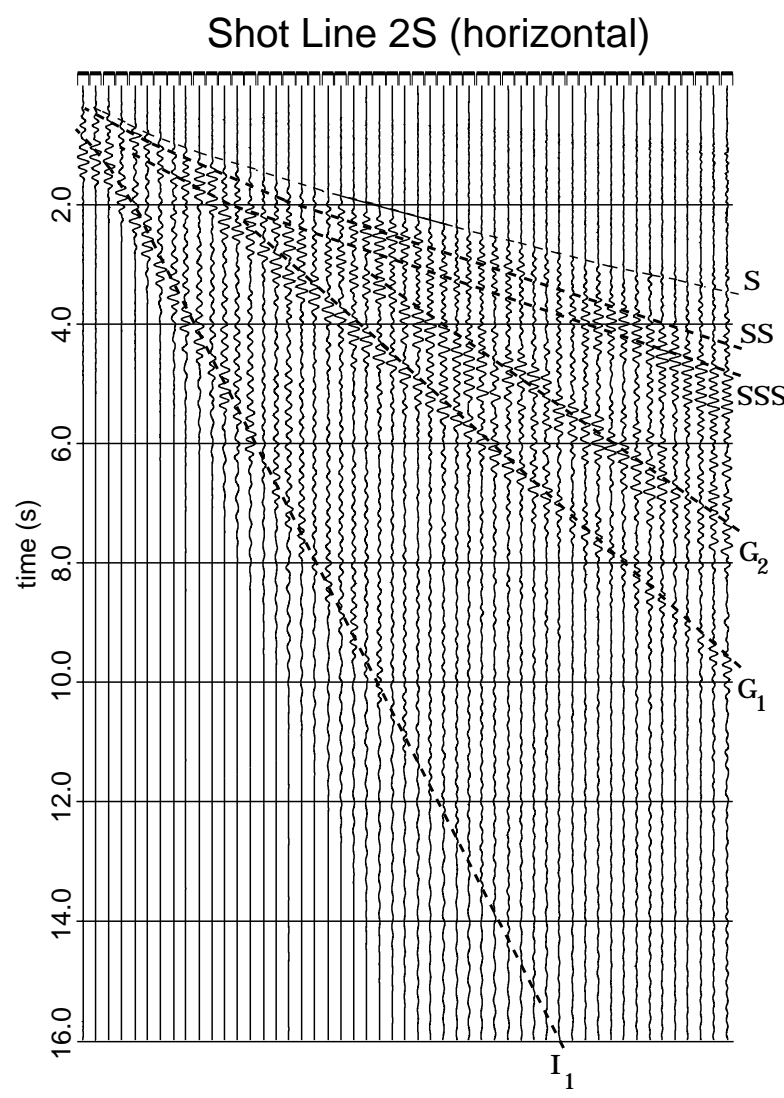

$8 \mathrm{~Hz}$ low-pass

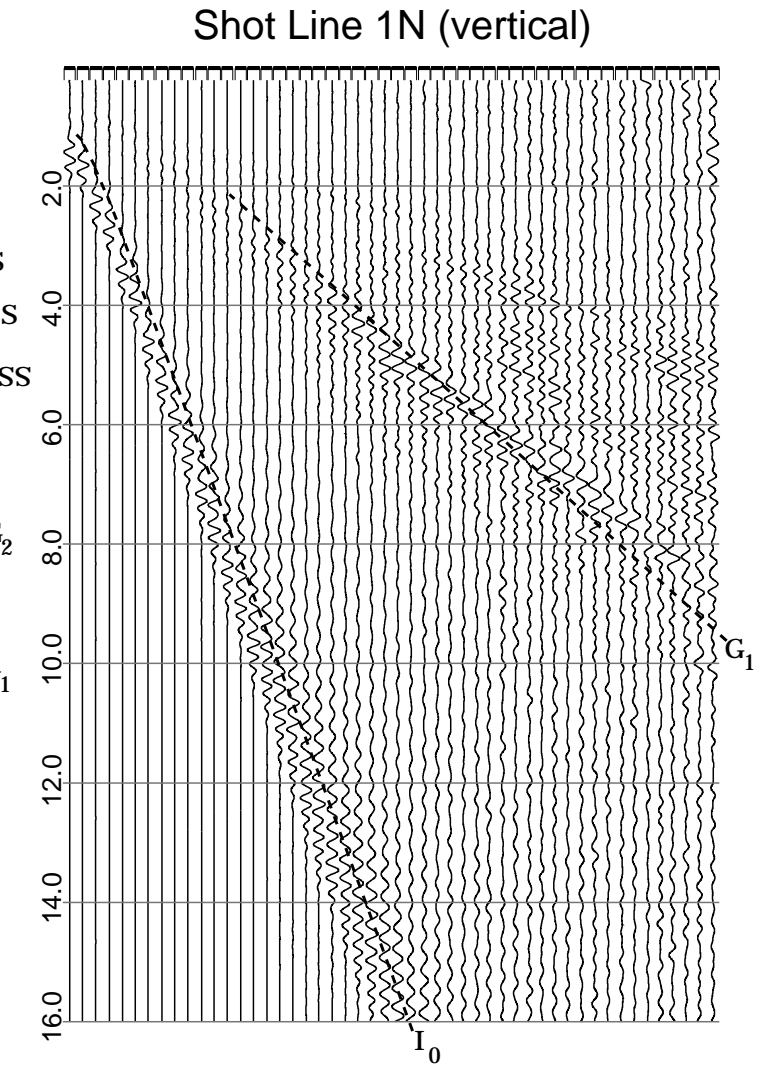

$5 \mathrm{~Hz}$ low-pass

FIG. 10. Marine data from Fairfield Industries. Water depth is about $5 \mathrm{~m}$ and acoustic sources are about $3 \mathrm{~m}$ above the sea floor. Vertical (top) and horizontal (bottom) component receiver gathers are from shot line $1 \mathrm{~N}$ for the vertical component and shot line $2 \mathrm{~S}$ for the horizontal component (see Fig. 9). Dashed lines on the vertical records are approximate arrival times of the fundamental Rayleigh wave $\left(I_{0}\right)$ and the first guided wave $\left(G_{1}\right)$ and on the horizontal records they are the refracted shear phase $S$, the refacted surface bounce phases $S S$ and $S S S$, and the group arrival times of the first Rayleigh overtones $\left(I_{1}\right)$ and two guided waves $\left(G_{1}, G_{2}\right)$. Shots range from about $50 \mathrm{~m}$ to about $1.2 \mathrm{~km}$ from the associated receiver. The vertical and horizontal records are low pass filtered with a high frequency corner at $5 \mathrm{~Hz}$ and $8 \mathrm{~Hz}$, respectively, to accentuate these low frequency shear phases. 

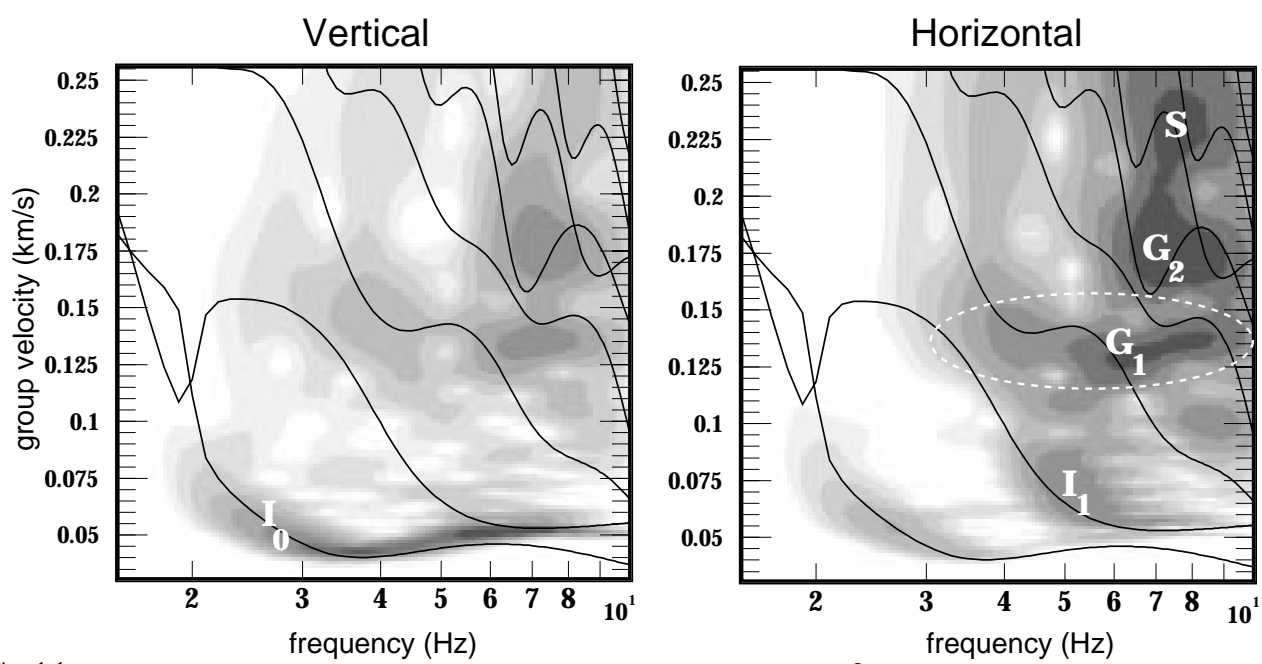

FIG. 11. Example of frequency-time diagrams for the Fairfield Industries data: record FF230

(Fig. 10) is from receiver gather $2 \mathrm{~S}$ (Fig. 9). The vertical component is at left and a horizontal component at right. Theoretical group velocity curves (Fig. 3a) computed from the shallow marine model (Fig. 17) are overplotted. Wave types are indicated. The shot-receiver distance is about 500 m. 


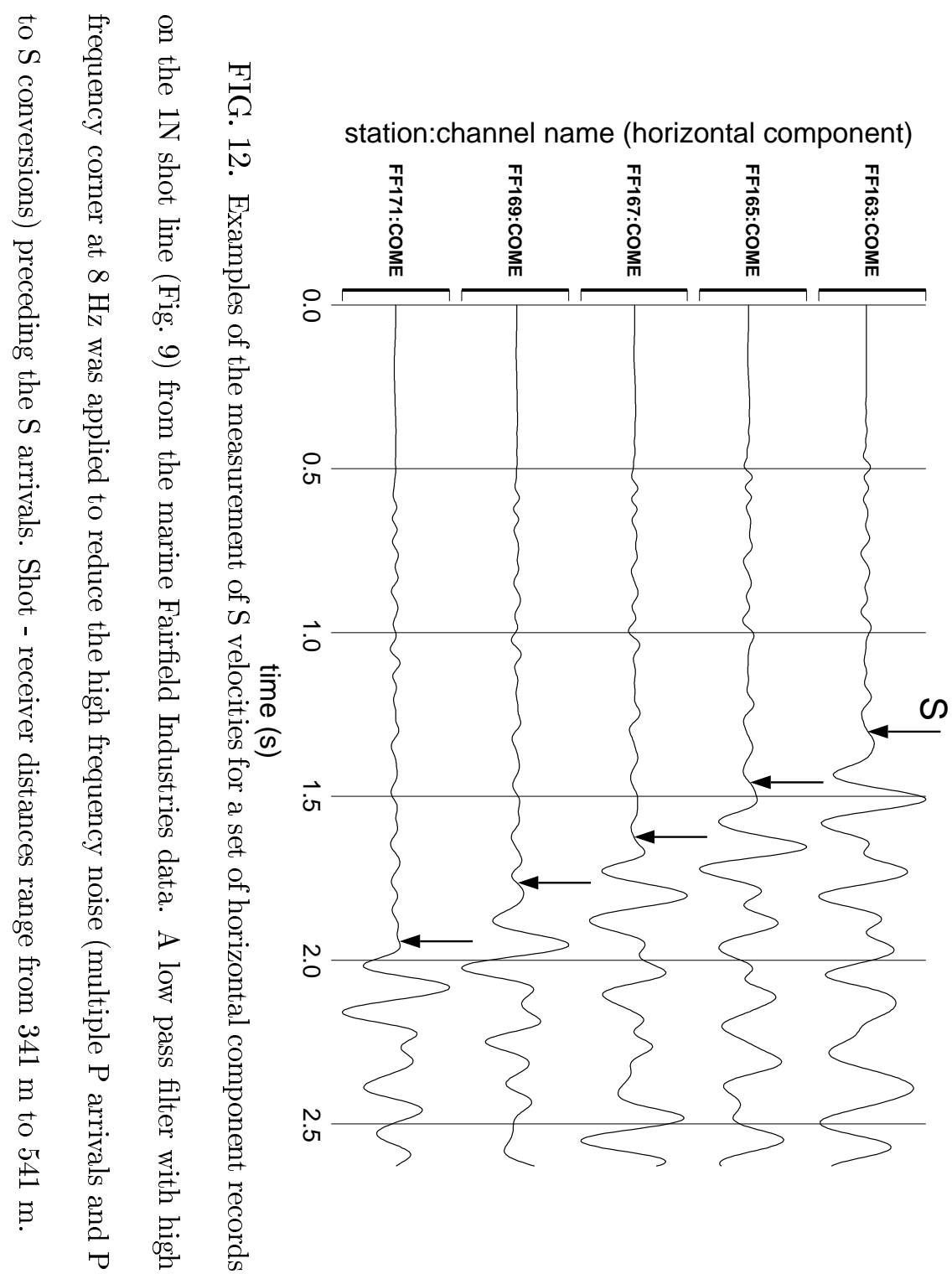


(a)

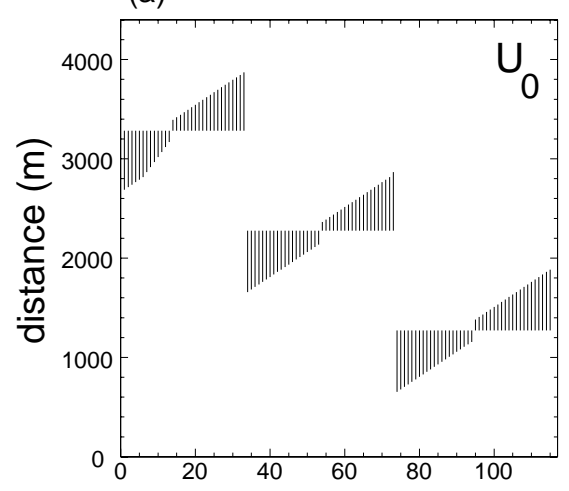

(b)

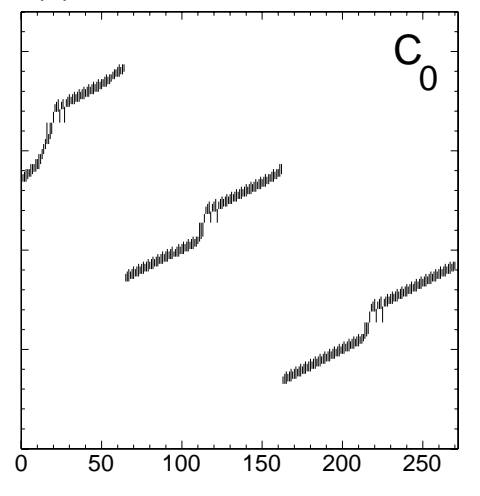

(c)

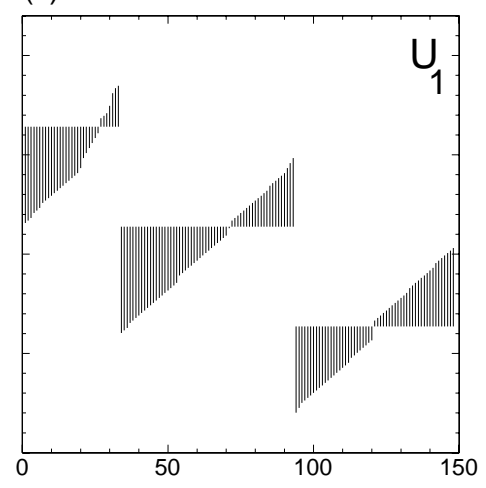

FIG. 13. Location of the wave paths of the dispersion measurements obtained from the Fairfield

Industries data: (a) fundamental Rayleigh wave $\left(I_{0}\right)$ group velocity, (b) fundamental Rayleigh wave phase velocity, and (c) first overtone Rayleigh wave $\left(I_{1}\right)$ group velocity. The vertical scales are identical to that in Fig. 9 and the horizontal scales indicate the measurement number. The wave paths of 115, 270 and 148 separate measurements are shown in panels (a) - (c), respectively. 

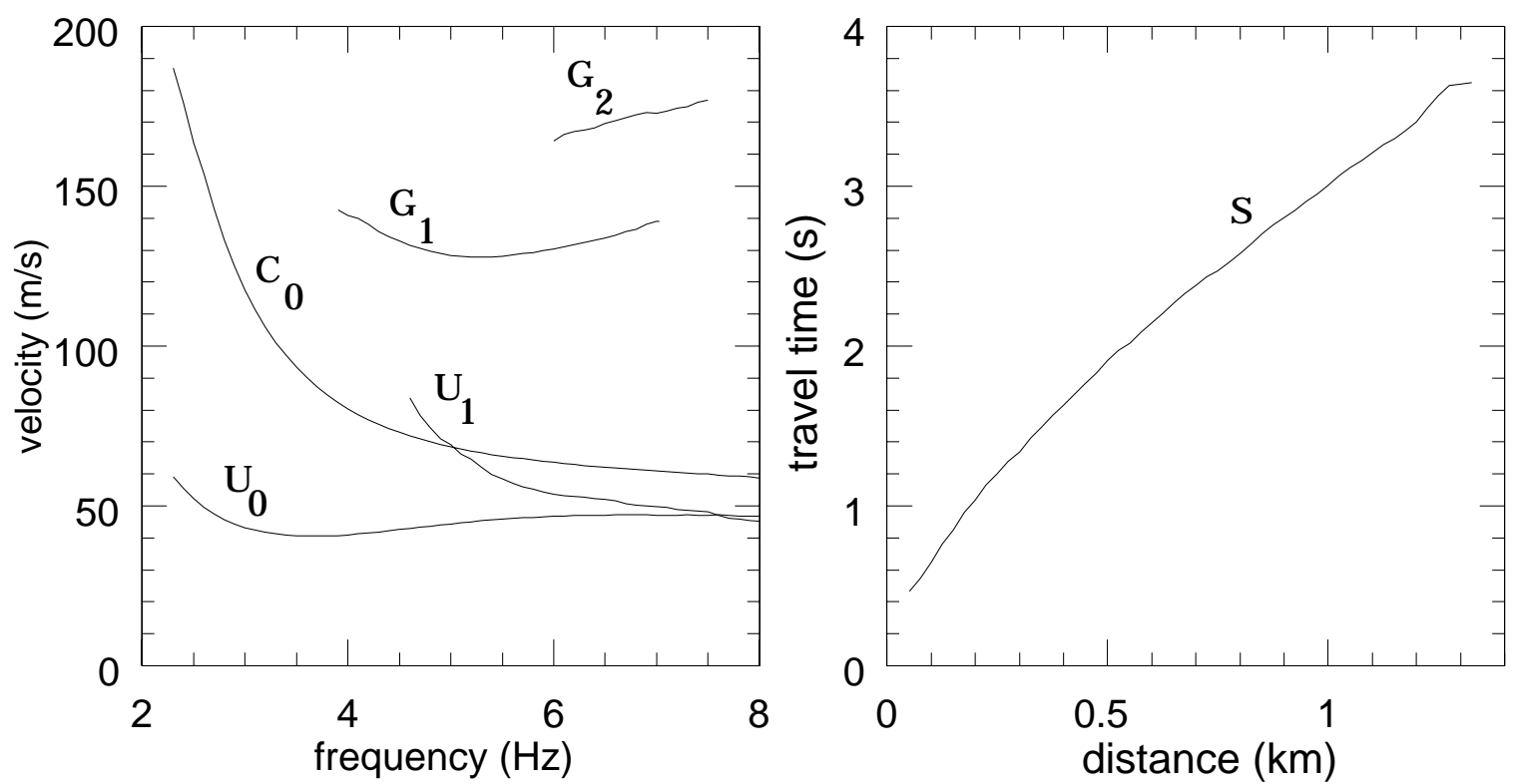

FIG. 14. Average of interface and guided wave dispersion and $S$ wave travel time measurements obtained from the Fairfield Industries data. (Left) Fundamental mode $\left(U_{0}\right)$, first overtone $\left(U_{1}\right)$, and guided wave $\left(G_{1}, G_{2}\right)$ group velocities and the phase velocity of the fundamental $\left(C_{0}\right)$ are shown. (Right) Average $S$ wave travel time. 


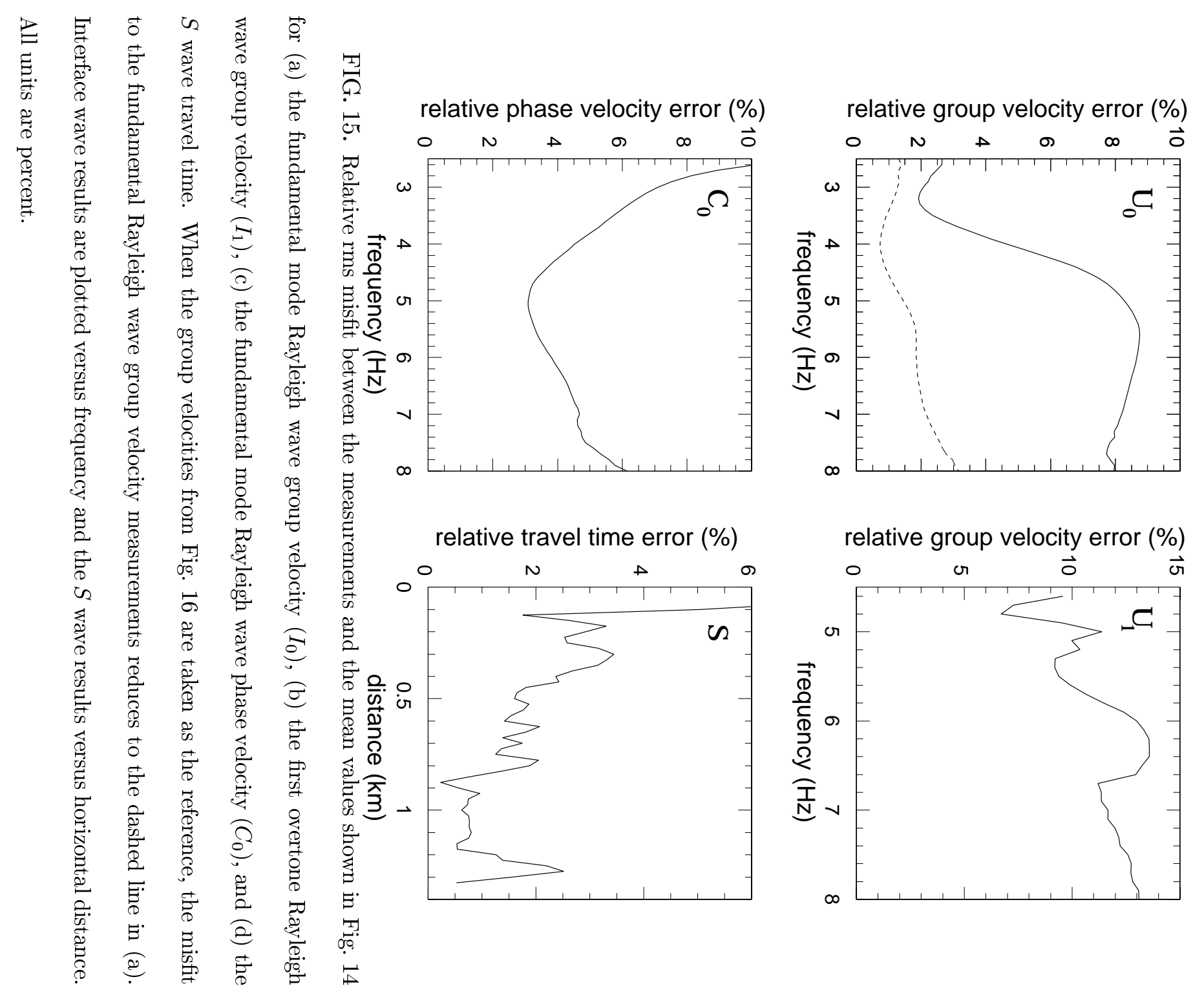



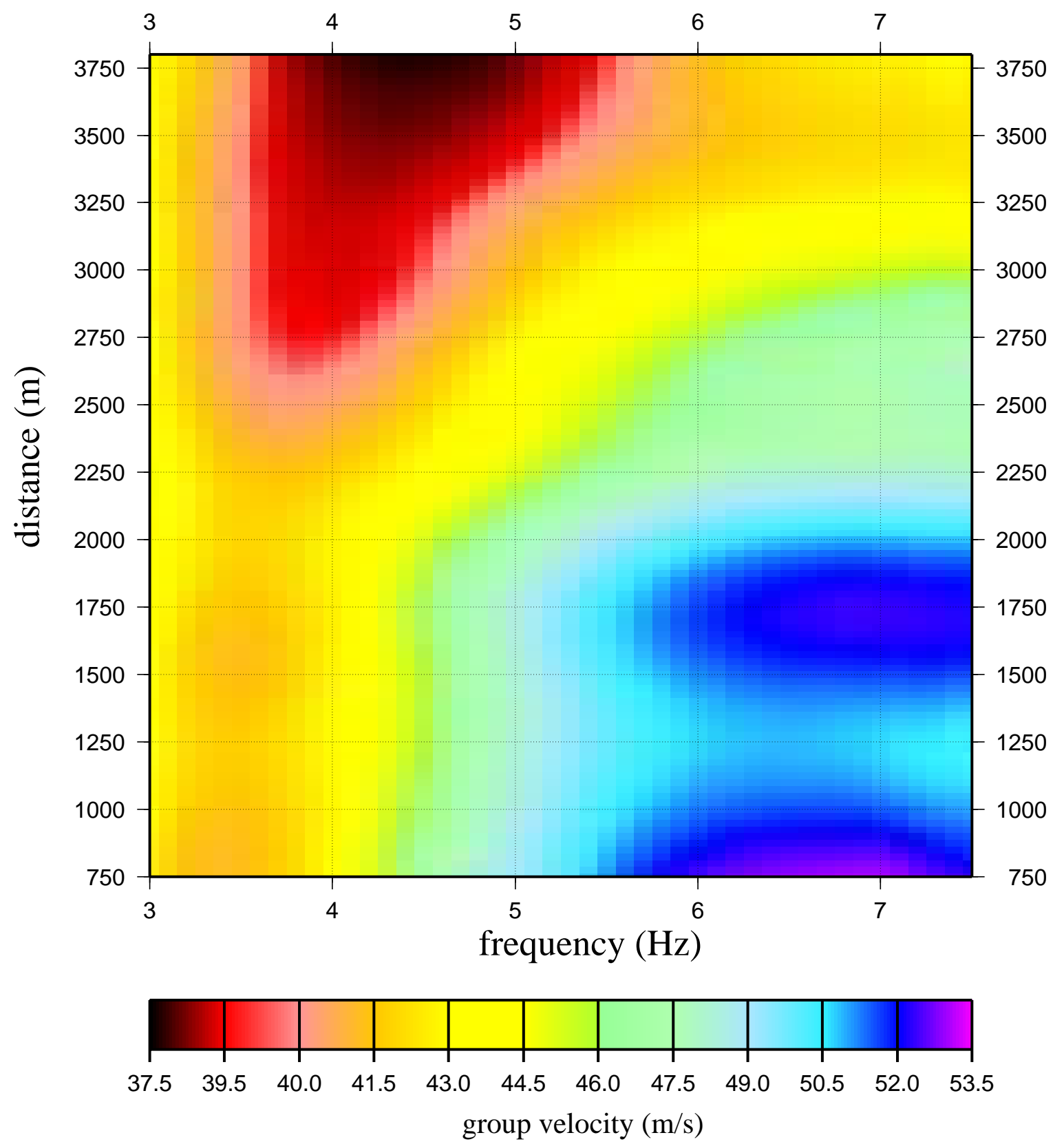

FIG. 16. Spatial variation of group velocity dispersion for the fundamental Rayleigh wave $\left(I_{0}\right)$ from the marine Fairfield Industries data. Distances refer to the spatial scale in Fig. 9. 
(a)

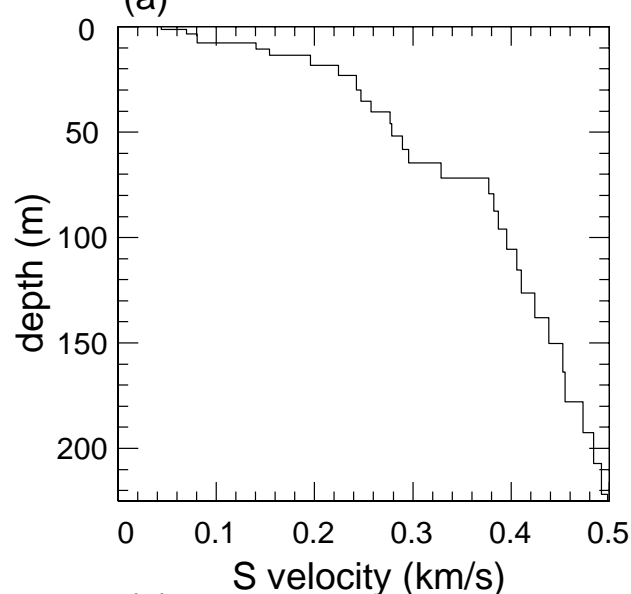

(b)

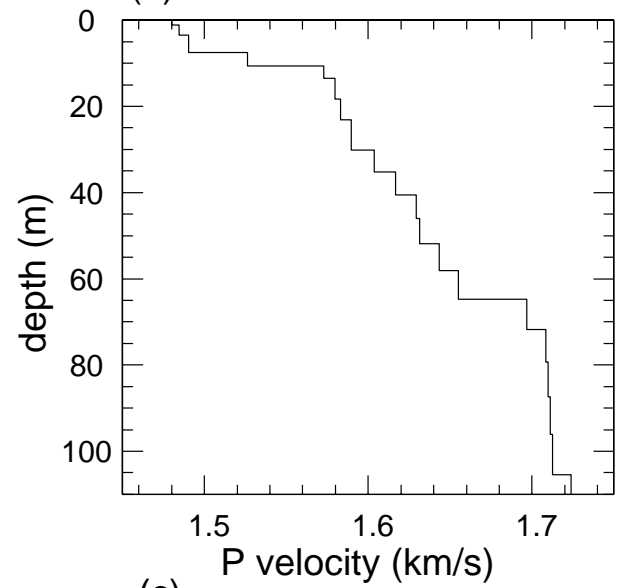

(c)

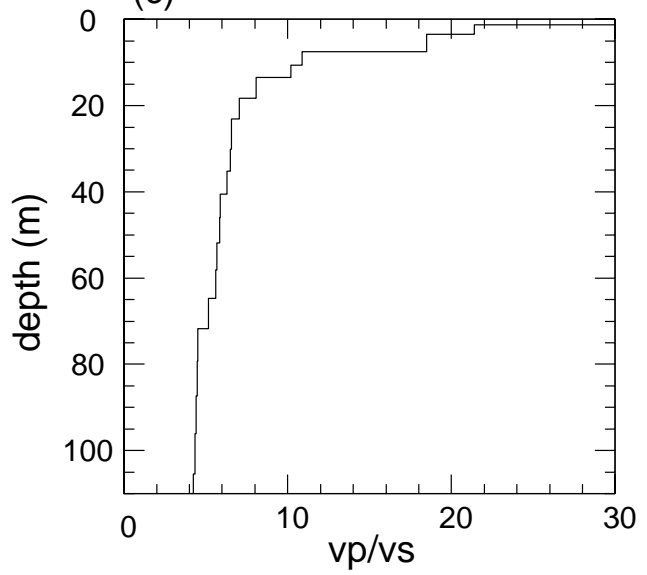

FIG. 17. One of the shallow marine models that fit the average composite data set shown in Fig. 14: (a) shear velocity $V_{s}$, (b) compressional velocity $V_{p}$, and (c) $V_{p} / V_{s}$. This is the model used throughout this paper. 

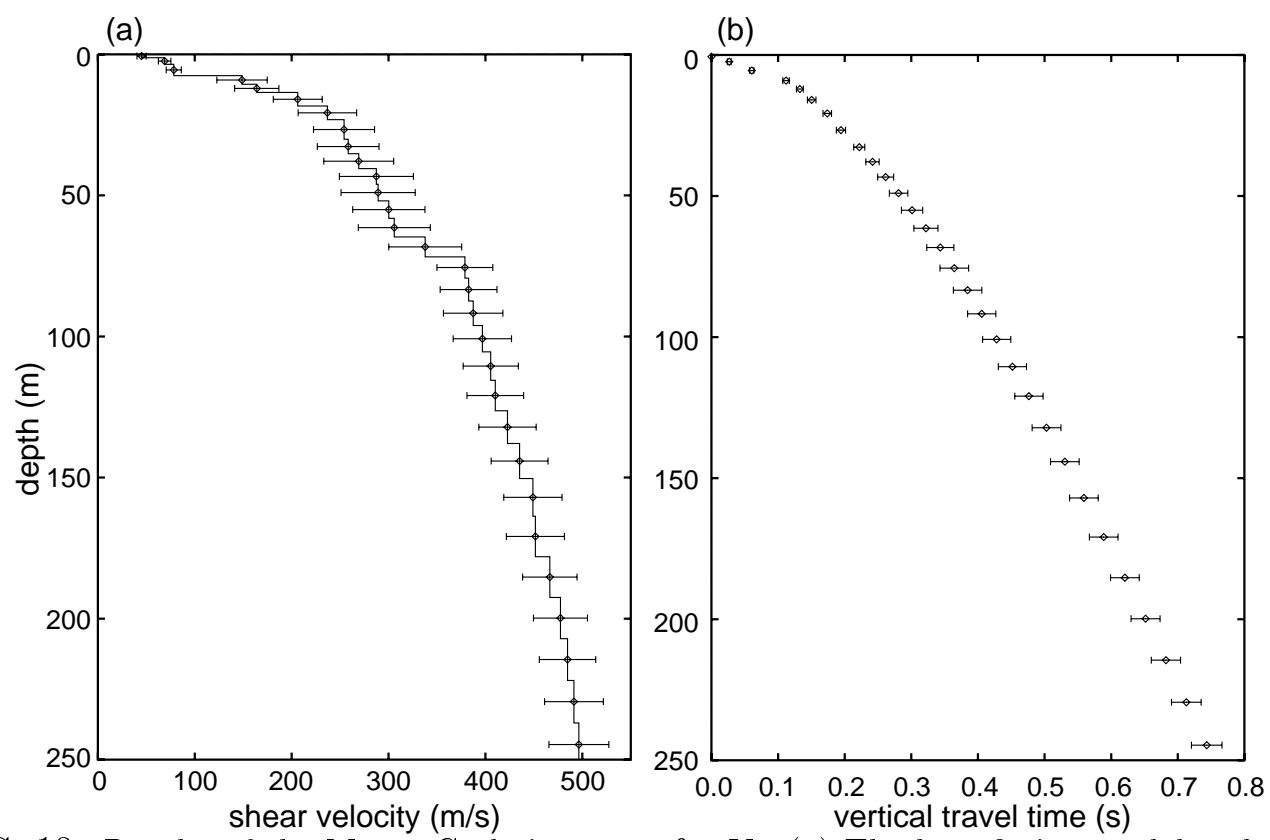

FIG. 18. Results of the Monte-Carlo inversion for $V_{s}$. (a) The best fitting model and $2 \sigma$ error bars, where $\sigma$ is the standard deviation in each layer of the ensemble of acceptable models. The best

fitting model is shown as the solid line. (b) Vertical shear wave travel time to the surface computed from each depth and $2 \sigma$ error bars, where $\sigma$ is the standard deviation of the travel time for the ensemble of acceptable models. 


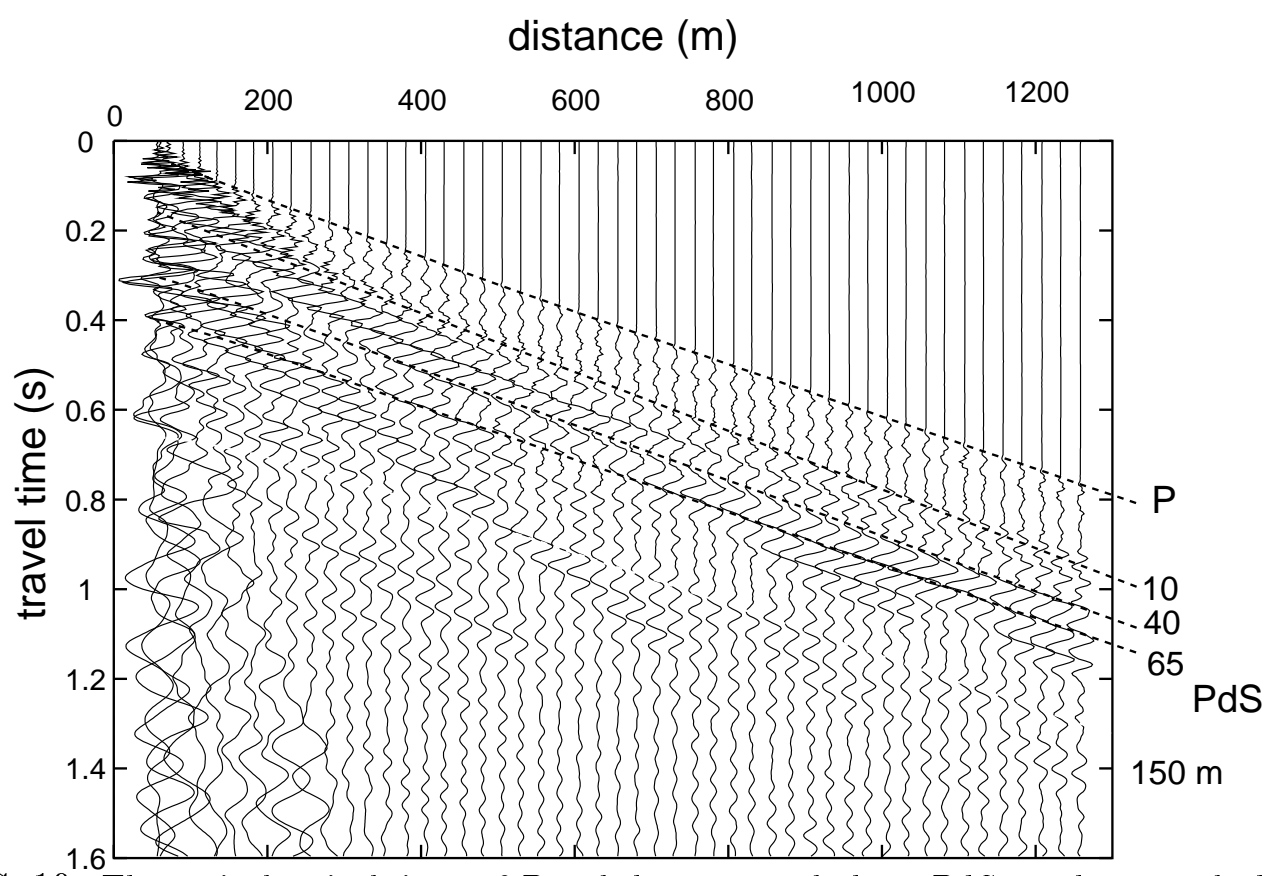

FIG. 19. Theoretical arrival times of $P$ and the converted phase $P d S$ are shown as dashed lines plotted over horizontal waveforms from the $2 \mathrm{~N}$ receiver gather (Fig. 9). The arrivals are computed with the shallow marine model shown in Fig. 17. Arrivals from different hypothesized reflectors are labeled with the depth of the reflector at the top of the figure. The amplitudes of the waveforms are modified by a geometrical spreading factor to facilitate comparison. 


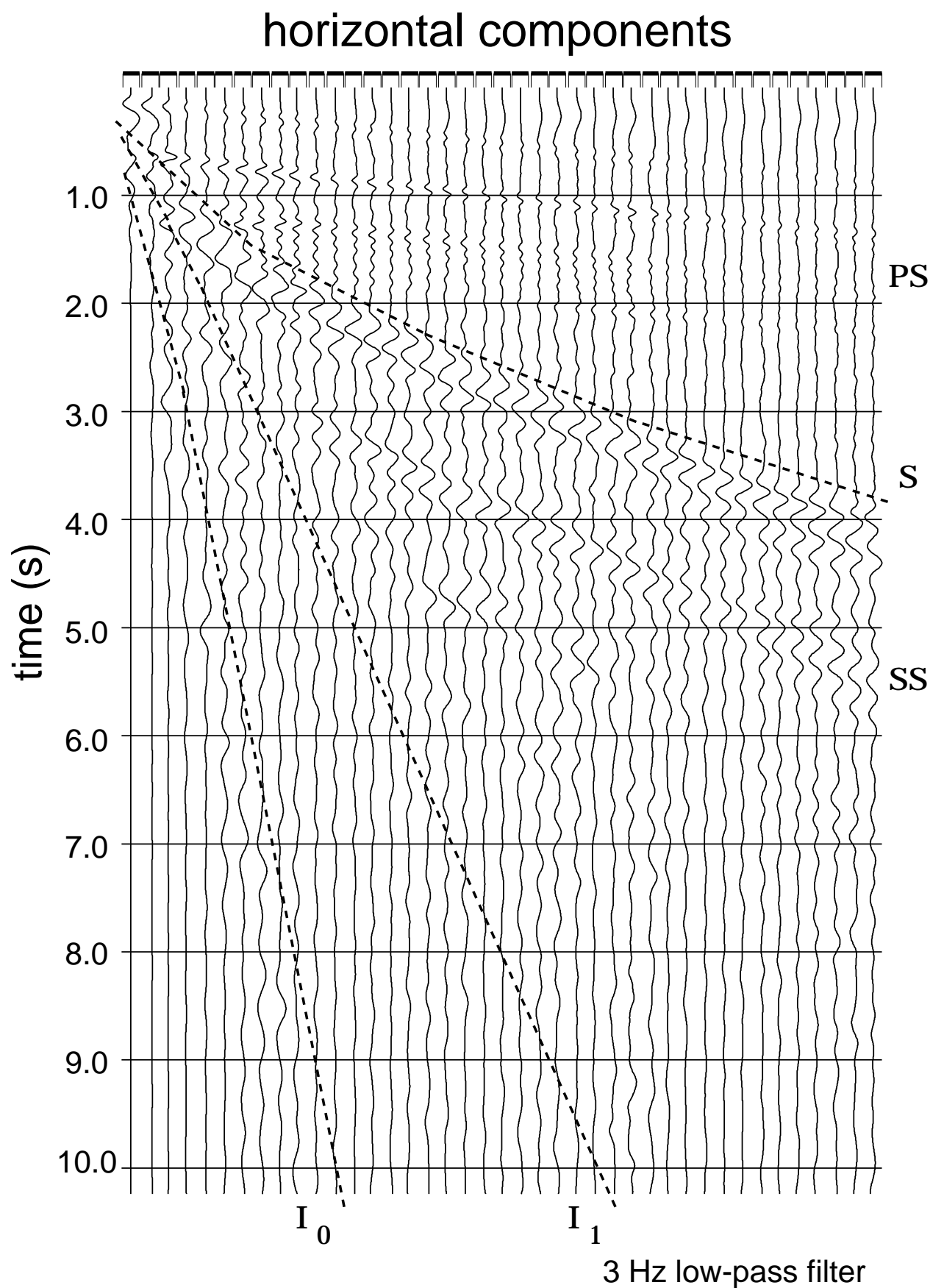

FIG. 20. Marine data from Western Geophysical. Water depth is slightly greater than $20 \mathrm{~m}$ on average and acoustic sources are about $20 \mathrm{~m}$ above the ocean bottom. Dashed lines represent approximate arrival times of $I_{0}, I_{1}$, and $S$. Shot WS2235 is about $32 \mathrm{~m}$ from the receiver and shot WS2275 is about $1.025 \mathrm{~km}$. All records have been low pass filtered with a high frequency corner at 3Hz. All records are horizontal components. 

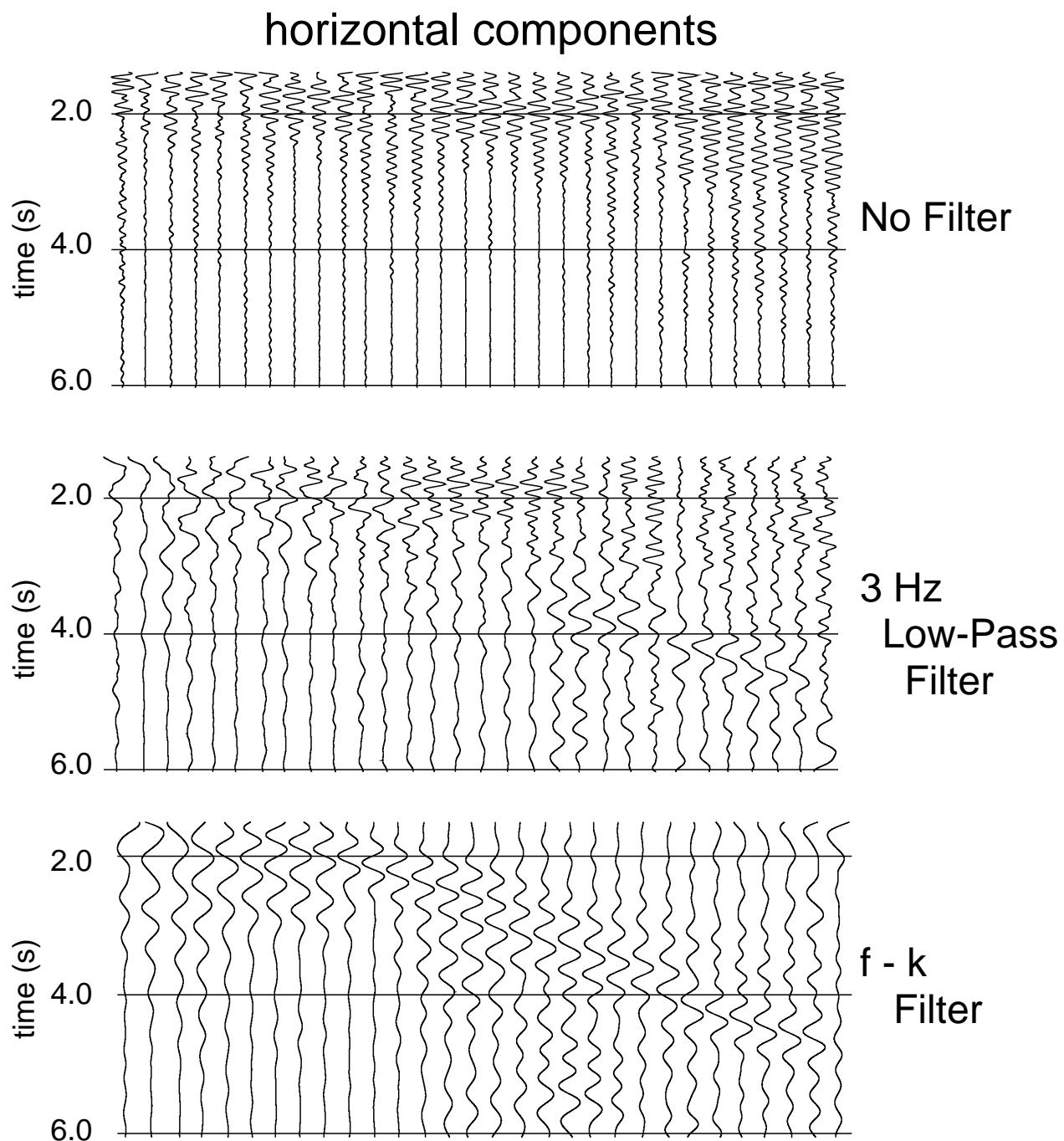

FIG. 21. Marine data from Unocal: (a) unfiltered, (b) low pass frequency filtered (high frequency corner at $3 \mathrm{~Hz}$ ), and (c) frequency-wavenumber filtered. Water depth is about $50 \mathrm{~m}$ and the acoustic source is about the same height above the ocean bottom. Refracted shear waves, guided waves, and interface waves are not seen on the raw data, but very low frequency shingled guided waves are barely visible on low pass filtered record sections. The guided waves, probably from the first overtone, are more clearly seen on records that have been filtered in the frequency-wavenumber domain. Shot spacing is about $25 \mathrm{~m}$ and source-receiver distances here range from $\sim 0$ to $\sim 750 \mathrm{~m}$. 